\title{
Myogenic Cell Expression of Intercellular Adhesion Molecule-1 Contributes to Muscle Regeneration after Injury
}

Ryan A. Martin, ${ }^{*}$ Kole H. Buckley, ${ }^{*}$ Drew C. Mankowski, ${ }^{*}$ Benjamin M. Riley, ${ }^{*}$ Alena N. Sidwell, ${ }^{*}$ Stephanie L. Douglas, ${ }^{*}$ Randall G. Worth, ${ }^{\dagger}$ and Francis X. Pizza*

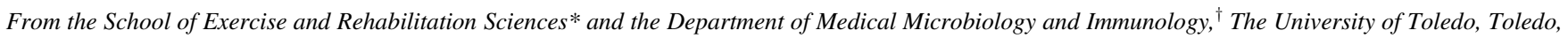
Ohio

\author{
Accepted for publication \\ June 15, 2020. \\ Address correspondence to \\ Francis X. Pizza, Ph.D., School \\ of Exercise and Rehabilitation \\ Sciences, The University of \\ Toledo, 2801 W. Bancroft St., \\ Toledo, $\mathrm{OH}$ 43606. E-mail: \\ francis.pizza@utoledo.edu.
}

\begin{abstract}
This study investigated intercellular adhesion molecule-1 (ICAM-1), a membrane protein that mediates cell-to-cell adhesion and communication, as a mechanism through which the inflammatory response facilitates muscle regeneration after injury. Toxin-induced muscle injury to tibialis anterior muscles of wild-type mice caused ICAM-1 to be expressed by a population of satellite cells/myoblasts and myofibers. Myogenic cell expression of ICAM-1 contributed to the restoration of muscle structure after injury, as regenerating myofibers were more abundant and myofiber size was larger for wild-type compared with Icam $1^{-1-}$ mice during 28 days of recovery. Contrastingly, restoration of muscle function after injury was similar between the genotypes. ICAM-1 facilitated the restoration of muscle structure after injury through mechanisms involving the regulation of myofiber branching, protein synthesis, and the organization of nuclei within myofibers after myogenic cell fusion. These findings provide support for a paradigm in which ICAM-1 expressed by myogenic cells after muscle injury augments their adhesive and fusogenic properties, which, in turn, facilitates regenerative and hypertrophic processes that restore structure to injured muscle. (Am J Pathol 2020, 190: 2039-2055; https://doi.org/10.1016/ j.ajpath.2020.06.009)
\end{abstract}

Skeletal muscle injury, resulting from physical activity or trauma, elicits a local inflammatory response, which contributes to the resolution of the injury by promoting processes of muscle regeneration. ${ }^{1-4}$ Indeed, components of the inflammatory response, such as macrophages, cytokines, and adhesion molecules, facilitate regenerative and hypertrophic processes within skeletal muscle by promoting proliferation of muscle precursor cells called satellite cells/myoblasts, ${ }^{4}$ as well as the formation and hypertrophy of myofibers. ${ }^{5-15}$ The release of cytokines (eg, insulin-like growth factor 1 and IL-6) and other molecules (eg, nitric oxide and a disintegrin and metalloproteinase with thrombospondin motifs 1) from macrophages serves as mechanisms through which they promote satellite cell/myoblast proliferation after injury., ${ }^{6,11,12,15}$ Little, however, is known about how the local inflammatory response regulates processes of muscle regeneration that are downstream of satellite cell/myoblast proliferation, such as homotypic adhesion and subsequent fusion of myogenic cells and protein synthesis, which are essential processes in the formation and hypertrophy of myofibers after injury. ${ }^{16,17}$

We have investigated intercellular adhesion molecule-1 (ICAM-1; CD54), which mediates cell-to-cell adhesion and communication during an inflammatory response, ${ }^{18,19}$ as a mechanism through which the inflammatory response facilitates regenerative and hypertrophic processes within skeletal muscle. ${ }^{13,20-22}$ Endothelial cells and leukocytes constitutively express ICAM-1 at low levels, and other cell types (eg, myogenic cells) express ICAM-1 after in vitro exposure to

Supported by a National Institute of Arthritis and Musculoskeletal and Skin Diseases of the NIH grant (F.X.P.); an American College of Sports Medicine grant (R.A.M.); and a Heart, Lung, and Blood Institute of the NIH grant (R.G.W.).

Disclosures: None declared. 
cytokines (eg, tumor necrosis factor- $\alpha$ and interferon$\gamma){ }^{13,23-26}$ Although satellite cells/myoblasts and myofibers do not normally express ICAM- $1,{ }^{13,20-26}$ myogenic cell expression of ICAM-1 has been reported in patients with inflammatory myopathies ${ }^{27-29}$ and in murine models of skeletal muscle hypertrophy ${ }^{13}$ and Duchenne muscular dystrophy. ${ }^{20}$ The extent to which myogenic cells express ICAM-1 after muscle injury remains to be determined. Myogenic cell expression of ICAM-1 after injury could facilitate the ensuing regeneration, as ICAM- 1 contributes to regenerative and hypertrophic processes after muscle overload ${ }^{13}$ and augments adhesive and fusogenic properties of myogenic cells in vitro. ${ }^{21,22}$

Our initial objective was to test the hypothesis that muscle injury induces ICAM-1 expression by satellite cells/myoblasts and myofibers. As myogenic cells were found to express ICAM-1 after muscle injury, we also tested the hypothesis that ICAM-1 contributes to the restoration of muscle structure and function after injury through a mechanism involving protein synthesis and myogenic cell fusion. This was accomplished by quantifying indexes of muscle structure, function, protein synthesis, and myogenic cell fusion (myofiber branching and myonuclear accretion) in wild-type and Icaml ${ }^{-1-}$ mice after toxin-induced muscle injury.

\section{Materials and Methods}

\section{Animals}

An equal number of male and female wild-type (C57BL/6) and $\operatorname{Icam} 1^{-1-}\left(\operatorname{Icam} 1 \mathrm{tm} 1^{\mathrm{Cws}}\right)^{30}$ mice were tested at 16 weeks of age. The Icaml ${ }^{-1-}$ mice were generated on a C57BL/6 background, ${ }^{30}$ and heterozygote littermates were not used as controls. Breeding colonies of wild-type and Icam $1^{-1-}$ mice were maintained separately after crossing and establishing their genotype.

Homozygous Pax7CreER2 $2^{31}$ and Rosa26-nT-nG ${ }^{32}$ mice were obtained from Jackson Laboratory (017763 and 023537, respectively; Bar Harbor, ME) and crossed. Pax $7^{\mathrm{CreER} 2 /+}$; $n T n G^{L o x P /+}$ mice at 12 weeks of age were injected intraperitoneally with tamoxifen in sunflower seed oil $(200 \mathrm{mg} / \mathrm{kg}$ per day) for 5 consecutive days, followed by a 2 -week washout period to induce nuclear localization of green fluorescent protein (GFP) in Pax $7^{+}$cells and their progeny.

Mice were housed and bred in an animal facility at The University of Toledo, which is accredited by the Association for the Assessment and Accreditation of Laboratory Animal Care. Mice were exposed to a 12-hour light-dark cycle and fed standard laboratory chow and water ad libitum. Mice were anesthetized using $2.5 \%$ isoflurane for all surgical procedures and were sacrificed via cervical dislocation. All procedures were approved by the Institutional Animal Care and Use Committee.

\section{Muscle Injury}

Muscle injury to tibialis anterior muscles was achieved by bilateral i.m. injection (50 $\mu \mathrm{L} /$ muscle) of $1.2 \% \mathrm{BaCl}_{2}$
(Sigma Aldrich, St. Louis, MO) in sterile water. A 25-gauge needle was inserted just above the distal tendon of tibialis anterior muscles and advanced proximally within the muscle belly until the needle spanned approximately $75 \%$ of the muscle length. The content of the syringe was dispensed, the needle was retracted, and skin incisions were sutured closed. Muscles were collected at 4, 7, 14, 21, and 28 days after injury, as well as from control mice, which did not undergo injury ( 0 days after injury).

\section{Western Blot Analysis}

Tibialis anterior muscles were homogenized in reducing sample buffer [2\% SDS, $50 \mathrm{mmol} / \mathrm{L}$ tris(2-carboxyethyl) phosphine hydrochloride, $80 \mathrm{mmol} / \mathrm{L}$ Tris-HCL, and $10 \%$ glycerol] containing protease inhibitors (Cytoskeleton Inc., Denver, CO). Concentration of proteins within samples was quantified, ${ }^{33}$ and samples were boiled and separated on $10 \%$ SDS-PAGE gels.

Proteins in gels were transferred to vinyl membranes (Immobilon-FL; Millipore-Sigma, St. Louis, MO) using a semidry (for ICAM-1: $20 \mathrm{~V}$ for 1 hour) or a wet transfer (for puromycin: 90 minutes and $200 \mathrm{~mA}$ ) protocol in Towbin buffer containing $10 \%$ methanol. Membranes were blocked with Tris-buffered saline containing 50\% Odyssey blocking buffer (for ICAM-1) or 5\% powdered milk (for puromycin). Membranes were washed in Tris-buffered saline $/ 0.05 \%$ Tween-20 and incubated overnight at $4^{\circ} \mathrm{C}$ with an antibody for ICAM-1 (R\&D Systems, Minneapolis, MN; AF796; 1:500), puromycin (Millipore; MABE343; 1:2500), ${ }^{34,35}$ or glyceraldehyde-3-phosphate dehydrogenase (loading control; Cell Signaling Technology, Danvers MA; 2118; 1:8000). Additional controls for sample loading included staining for total protein using Krypton (for membranes; ThermoFisher, Ann Arbor, MI) or Coomassie Blue (for gels). Detection of bound primary antibodies was achieved using species or isotypespecific Alexa Fluor 680 secondary antibody (1:5000; Invitrogen, Carlsbad, CA). Detection and quantification of ICAM1 and puromycin were performed using the Odyssey infrared detection system.

\section{Immunofluorescence}

Transverse sections ( $10 \mu \mathrm{m}$ thick) from the midbelly of muscles were fixed in cold acetone, treated with blocking buffer ( $10 \%$ fetal bovine serum or $3 \%$ bovine serum albumin, $0.05 \%$ Tween-20, and $0.2 \%$ gelatin in phosphate-buffered saline), and incubated with one or more of the following antibodies: goat anti-mouse ICAM-1 (R\&D Systems; AF796; 1:100), rabbit anti-dystrophin (which recognizes the C-terminus of dystrophin; ThermoFisher; RB-9024-P; 1:100), rabbit anti-desmin (Sigma-Aldrich; D8281; 1:75), and mouse anti-puromycin (Millipore; MABE343; 1:500). Bound primary antibodies were detected using Alexa Fluor 488 or 594 secondary antibodies (Jackson ImmunoResearch, West Grove, PA). Nuclei were stained using DAPI (SouthernBiotech, Birmingham, 
$\mathrm{AL})$. Sections were imaged using an epifluorescence microscope (Olympus IX70: B\&B Microscopes, Pittsburgh, PA) equipped with a charge-coupled device monochrome camera (RT KE SPOT; Diagnostic Instruments, Sterling Heights, MI).

Immunolabeling was performed on single myofibers isolated from tibialis anterior muscles fixed in formaldehyde, as described below (Single-Myofiber Analysis). Pilot work demonstrated that formaldehyde fixation inhibited detection of ICAM- 1 and that ICAM- 1 detection could be restored by bathing formaldehyde-fixed single myofibers in cold acetone. Thus, formaldehyde-fixed single myofibers were treated with cold acetone, $10 \%$ fetal bovine serum, and goat anti-mouse ICAM-1 (R\&D Systems; AF796; 1:100) and rabbit antidystrophin (ThermoFisher; RB-9024; 1:100) antibodies. Bound primary antibodies were detected using Alexa Fluor 488 or 594 secondary antibodies (Jackson ImmunoResearch), and nuclei were stained using DAPI (SouthernBiotech).

Immunolabeling was also performed on muscle sections of tibialis anterior muscles from tamoxifen-treated $\operatorname{Pax} 7^{\mathrm{C}-}$ reER2/+; nTnGLoxP/+ mice. Sections were treated with $4 \%$ formaldehyde, cold acetone, and blocking buffer (3\% bovine serum albumin, $0.05 \%$ Tween-20, and $0.2 \%$ gelatin in phosphate-buffered saline). Muscle sections were incubated with goat anti-mouse ICAM-1 (R\&D Systems; AF796; 1:100) and rabbit anti-GFP (Invitrogen; number A11122; 1:500) followed by Alexa Fluor 594 and 488 secondary antibodies (Jackson ImmunoResearch). Nuclei were stained using DAPI (SouthernBiotech). Images of ICAM-1 and GFP were captured using settings established from muscle sections treated with secondary, but not primary, antibodies. These settings produced no red fluorescence in antibody control slides from injured muscles.

\section{Isolation of Satellite Cells/Myoblasts}

Tibialis anterior muscles were excised, minced in Dulbecco's modified Eagle's medium, centrifuged, and incubated at $37^{\circ} \mathrm{C}$ in Dulbecco's modified Eagle's medium containing $1000 \mathrm{U} / \mathrm{mL}$ of collagenase IV (Worthington, Lakewood, NJ) for 60 minutes, followed by incubation with $100 \mathrm{U} / \mathrm{mL}$ collagenase IV and $1 \mathrm{U} / \mathrm{mL}$ dispase II (Worthington) for 30 minutes. Enzymatic digestion was performed at $37^{\circ} \mathrm{C}$ under continuous rotation, and the GentleMACS Dissociator (Miltenyl Biotec, Bergisch Gladbach, Germany) was used at 30, 60 , and 90 minutes of digestion. Cell suspensions were filtered (60 $\mu \mathrm{m}$; Millipore), centrifuged, and suspended in staining buffer.

Cells were incubated with biotin-conjugated antibodies (BD Biosciences, East Rutherford, NJ) to detect leukocytes (CD45 and CD11b), endothelial cells (CD31), and multipotent hematopoietic stem cells (Sca-1; Ly-6A/E) followed by an anti-biotin-phycoerythrin/phosphatidylethanolamine (SouthernBiotech) secondary antibody. Fluorophoreconjugated antibodies were used to identify ICAM-1 [CD54-allophycocyanin (APC)]; Molecular Probes, Eugene, OR) and integrin $\alpha 7$ (integrin $\alpha 7-$ Alexa Fluor 488;
AbLab, Point Grey, BC, Canada). Cells were analyzed via flow cytometry (FACSCalibur; BD Biosciences) using isotype control antibodies for APC, Alexa Fluor 488, and phosphatidylethanolamine, and standard gating procedures. Satellite cells/myoblasts were defined as cells that were negative for CD45/CD11b/CD31/Sca-1 and positive for $\alpha 7$ (Lin ${ }^{-} \alpha 7^{+}$cells). ${ }^{36,37}$ Satellite cell/myoblast expression of ICAM-1 is reported as a percentage of the total $\operatorname{Lin}^{-} \alpha 7^{+}$ population and as the number of ICAM- $1^{+} \operatorname{Lin}^{-} \alpha 7^{+}$cells expressed relative to muscle mass (mg).

\section{Histology}

Myofiber size and regenerating myofiber number were determined after delineating their membrane and surrounding connective tissue via wheat germ agglutinin staining. Muscle sections (10 $\mu \mathrm{m}$ thick) were fixed in $4 \%$ formaldehyde, washed in phosphate-buffered saline, and incubated for 60 minutes with Alexa Fluor 555 conjugated wheat germ agglutinin (ThermoFisher; $10 \mu \mathrm{g} / \mathrm{mL}$ ), which binds to components of connective tissue (eg, $\mathrm{N}$-acetylglucosaminyl residues) and membrane structures (eg, glycoproteins). Nuclei were stained using DAPI (SouthernBiotech). Six to eight fields of view per muscle section were imaged via epifluorescence microscopy.

The cross-sectional area of myofibers and the number of regenerating myofibers were quantified using image analysis software (Image Pro 7; Media Cybernetics, Rockville, MD). Briefly, red (wheat germ agglutinin) and blue (nuclei) were extracted from a merged image to generate separate luminance images. An outline of myofibers that had an area $>200 \mu \mathrm{m}^{2}$ was generated and merged with the image of nuclei. The area of outlines/myofibers and the number of nuclei within and not in contact with the outline were quantified. On average, 556 myofibers per muscle (SD $= \pm 222$ myofibers) and 4437 myofibers per group (SD $= \pm 863$ myofibers) were analyzed $(n=8$ muscles per group).

The average cross-sectional area of myofibers within a muscle was used to make statistical comparisons between the genotypes. Myofibers with one or more centrally located nuclei were defined as regenerating myofibers. The percentage of regenerating myofibers within a muscle was calculated using the following equation: (number of myofibers with central nucleation/total number of myofibers analyzed) $\times 100$.

\section{Muscle Function}

Peak isometric torque was measured in tibialis anterior muscles using procedures that were similar to those used by others. ${ }^{38,39}$ Briefly, anesthetized mice were placed on a temperature-controlled platform. The knee was clamped and the foot was taped to a footplate attached to the shaft of a servomotor (Aurora Scientific, Aurora, ON, Canada; model 305B). Needle electrodes were inserted into the skin around 
the knee to stimulate the common peroneal nerve. Optimal electrode placement, voltage ( 5 to $9 \mathrm{~V}$ ), and frequency (150 to $300 \mathrm{~Hz}$ ) were determined within 5 to 15 isometric contractions using pulse-train stimulation (200-millisecond train of 0.2-millisecond pulses). Peak isometric torque of tibialis anterior muscles was determined after tenotomy of extensor digitorum longus and extensor hallicus longus muscles.

\section{Protein Synthesis}

Protein synthesis was measured in tibialis anterior muscles using the surface sensing of translation technique. ${ }^{34,35}$ Mice were anesthetized using isoflurane and injected intraperitoneally with puromycin $(0.4 \mu \mathrm{mol} / \mathrm{g}$ body mass $)$. Mice remained anesthetized for 30 minutes, at which time tibialis anterior muscles were surgically removed, frozen, and stored at $-80^{\circ} \mathrm{C}$. Puromycin incorporation into proteins was detected via Western blot analysis and immunofluorescence, as described above. Quantification of puromycin incorporation into proteins in Western blots was performed over the entire lane. ${ }^{35}$

Puromycin incorporation into proteins was also quantified using a slot blot approach. Samples were boiled, loaded into a Bio-Dot (Bio-Rad, Hercules, CA; $15 \mu \mathrm{g}$ per slot), and proteins within samples were immobilized on nitrocellulose membranes via vacuum filtration. Membranes were blocked using 5\% powdered milk, washed in Tris-buffered saline/ $0.05 \%$ Tween-20, and incubated overnight at $4{ }^{\circ} \mathrm{C}$ with a mouse anti-puromycin antibody (Millipore; MABE343; 1:5000) followed by an isotype-specific Alexa Fluor 680 secondary antibody. Ponceau staining of membranes served as controls for sample loading. Detection and quantification of puromycin was performed using the Odyssey infrared detection system.

\section{Single-Myofiber Analysis}

Hind limbs were skinned, removed, secured to stiff wires at 90-degree ankle flexion, and bathed in $4 \%$ formaldehyde for 2 days at $4{ }^{\circ} \mathrm{C}$ with constant mixing. Tibialis anterior muscles were excised and bathed in $40 \% \mathrm{NaOH}$ for 2 hours. ${ }^{40}$ Single myofibers were teased apart at the distal tendon using surgical forceps and transferred to slides coated with a solution containing $0.04 \%$ chromium (III) potassium sulfate and $0.4 \%$ gelatin. Nuclei within myofibers were stained with DAPI (SouthernBiotech), and formaldehyde-induced fluorescence was used to delineate myofibers. The entire length of myofibers was imaged using an automated imaging system (Olympus; VS120) at multiple z-planes that were separated by $1.3 \mu \mathrm{m}$.

Myofibers were examined for signs of branching. Myofibers that had a fissure that resembled a bridge or had a bifurcation on their side or at their end were scored as branched. ${ }^{41-44}$ On average, 23 myofibers per muscle (18 to 26 myofibers per muscle) were analyzed, and the percentage of myofibers that exhibited a branched morphology was calculated for each muscle ( $n=3$ muscles per group).

Quantitative analysis of myofiber size and number of nuclei was performed on z-plane images using cellSens (B\&B Microscopes) and Image Pro 7 (Media Cybernetics) software. One or two segments of a myofiber were analyzed for length and averaged width using cellSens. Regions of a myofiber that exhibited a branched morphology were included in this study's quantitative analysis, except for those that had a large fissure or a bifurcated side. Ends of myofibers were not analyzed. On average, $3939 \mu \mathrm{m}$ (SD $= \pm 1043 \mu \mathrm{m})$ of myofiber length was analyzed ( $n=393$ myofibers), which corresponded to $63 \%$ of the measured length of myofibers. Area of each myofiber segment analyzed was calculated $\left(\mathrm{mm}^{2}=\right.$ length $\times$ width $)$.

Myofiber segments that were measured for length and width were used in the analysis of nuclei. Given the proximity of nuclei within myofibers after injury, nuclei were separated from each other via watershed split before quantitative analysis using Image Pro 7. The number of nuclei, including those in a peripheral location near the outline of myofibers (ie, nuclei of presumptive satellite cells), the number of clusters of nuclei, and the number of nuclei within a cluster were quantified using Image Pro 7. Nuclei in contact with or outside of the outline of myofibers were not counted. A cluster of nuclei was defined as a nonlinear grouping of three or more nuclei that were not separated by watershed split. The length of linear arrays of nuclei, called nuclear chains, ${ }^{45}$ was measured using cellSens. A nuclear chain was defined as a series of $\geq 10$ nuclei that were organized in a linear array near the center of myofibers. Termination of chain length occurred when the spacing between nuclei within a chain reached approximately $60 \mu \mathrm{m}$ or when a nucleus within a chain was clustered with other nuclei.

Data for individual myofibers in each muscle were averaged (18 to 26 myofibers per muscle), and statistical comparisons between the genotypes were made using mean values for a muscle. Three muscles per group were statistically analyzed.

\section{Statistical Analysis}

Data sets were tested for normality and equal variance and analyzed using one- or two-way analysis of variance using Sigma Plot statistical software version 13 (Systat, Chicago, IL). Grouping factors for the two-way analysis of variance were genotype (wild type and Icaml ${ }^{-l-}$ ) and postinjury time point. The Newman-Keuls post-hoc test was then used to locate differences between groups when the observed $\mathrm{F}$ ratio was statistically significant $(P<0.05)$. Linear regression analysis was performed using Sigma Plot. Frequency distribution analyses were performed for descriptive purposes using Excel (Microsoft, Redmond, WA). Data analyzed via analysis of variance are reported as mean and SEM, whereas descriptive data are reported as mean and SD. 


\section{Results}

\section{ICAM-1 Expression after Muscle Injury}

Western blot analysis of muscle homogenates was performed to establish the temporal pattern of ICAM-1 expression after muscle injury (Figure 1A). Protein levels of ICAM-1 at 7 days after injury were ninefold higher than control levels $(P<0.001)$ (Figure 1B). At other recovery time points, protein levels of ICAM-1 were threefold to fourfold higher than control levels. As noted by the SEM bars in Figure 1B, the relative abundance of ICAM-1 varied between samples at each time point. The Western blot shown in Figure 1A was obtained during data collection, and samples were not re-analyzed to produce a Western blot for publication that matches the temporal pattern of the group data. The expression profile of ICAM-1 was temporally related to the established time course of satellite cell/ myoblast proliferation, as well as regenerating myofiber formation and hypertrophy after toxin-induced muscle injury. ${ }^{46}$

\section{Myofiber Expression of ICAM-1 after Muscle Injury}

Immunolabeling for ICAM-1 and dystrophin was performed to determine the extent to which ICAM-1 is expressed on the sarcolemma of myofibers after muscle injury (Figure 1C).

In control muscles, ICAM-1 was not colocalized with dystrophin, which is consistent with the evidence that myofibers do not constitutively express ICAM-1. ${ }^{13,20,27,29}$ At 7, 14, and 21 days after injury, ICAM-1 surrounded most myofibers. Much of the ICAM-1 surrounding myofibers at 4 and 7 days after injury was localized to cells residing in the interstitium. More important, myofibers after muscle injury expressed ICAM-1, as indicated by the colocalization of ICAM-1 and dystrophin, as well as an occasional detection of ICAM-1 in the sarcoplasm. ICAM-1 was also found localized to dystrophin in myofibers isolated from muscles after injury (Figure 2A). These observations indicate that muscle injury induces myofiber expression of ICAM-1 during muscle regeneration.

\section{Satellite Cells/Myoblasts Express ICAM-1 after Muscle Injury}

To explore the possibility that myoblasts express ICAM-1 after injury, muscle sections were surveyed for ICAM-1 expression by dystrophin ${ }^{+}$mononuclear cells ${ }^{47}$ and labeling was performed for ICAM-1 and desmin, a commonly used marker of myoblasts. ${ }^{48}$ Dystrophin $^{+}$(Figure 2B), as well as desmin $^{+}$(Figure 2C), mononuclear cells expressed ICAM-1 after muscle injury, particularly at 4 and 7 days after injury. Labeling could not be performed for ICAM-1 and Pax7, an established marker of satellite cells, ${ }^{49}$ as the optimal protocol for detecting Pax7, which included formaldehyde fixation and epitope retrieval, impaired detection of ICAM1. To circumvent this issue, a Pax7 nuclear reporter strain of mice $\left(\operatorname{Pax} 7^{\mathrm{CreER} 2 /+} ; n T n G^{\mathrm{LoxP} /+}\right)$ in which the nucleus of $\operatorname{Pax} 7^{+}$cells and their progeny indelibly express GFP after tamoxifen-induced recombination was used. At 4 and 7 days after injury, ICAM-1 was expressed by mononuclear cells expressing nuclear GFP (Figure 2D). Our analysis of muscle sections was interpreted to indicate satellite cell/myoblast expression of ICAM-1 after injury.

To quantitatively evaluate ICAM-1 expression by satellite cells/myoblasts, flow cytometry was performed on satellite cells/myoblasts isolated from tibialis anterior muscles. Satellite cells/myoblasts were defined as cells that did not express lineage markers (CD45/CD11b/CD31/Sca-1) and expressed integrin $\alpha 7$ (ie, $\operatorname{Lin}^{-} \alpha 7^{+}$cells) (Figure 2, E-G). ${ }^{36,37}$ Control muscles contained few to no ICAM-1 expressing satellite cells/myoblasts, which is consistent with the evidence that satellite cells/myoblasts do not constitutively express ICAM-1. ${ }^{13,21-25}$ The percentage of satellite cells/myoblasts expressing ICAM-1 (Figure 2F) and the number of ICAM- $1^{+}$satellite cells/myoblasts expressed relative to muscle mass (Figure $2 \mathrm{G}$ ) were elevated above control levels at 7 days after injury. The percentage and number of ICAM-1 ${ }^{+}$satellite cells/myoblasts at 14 days after injury were similar to levels observed in controls. These findings indicate that a small population of satellite cells/myoblasts express ICAM-1 after injury.

\section{ICAM-1 Expression by Neighboring Myogenic Cells}

Myogenic cells (dystrophin ${ }^{+}$or desmin $^{+}$) expressing ICAM-1 after injury were frequently found closely associated with myogenic cells that also expressed ICAM-1 (Figure 2, A and B, and Figure 3). Such occurrences were observed between presumptive satellite cells/myoblasts (dystrophin $^{+}$or desmin ${ }^{+}$mononuclear cells) (Figure 2, A and $\mathrm{B}$, and Figure $3 \mathrm{~A}$ ), satellite cells/myoblasts and myofibers (Figures 2A and 3B), and between myofibers (Figure 3C). In serial sections, neighboring myofibers that expressed ICAM-1 that appeared to be in the process of fusing together (Figure 3D) were observed. These observations were interpreted to indicate ICAM-1-ICAM-1 interactions between opposing myogenic cells (ie, homophilic trans interactions for ICAM-1) after muscle injury, which has been demonstrated to augment myogenic cell adhesion and subsequent fusion in vitro. ${ }^{21,22}$

\section{ICAM-1 Reduces Myofiber Branching after Injury}

Muscle regeneration after injury is accompanied by the formation of branched myofibers, ${ }^{42-44}$ which have a membrane and cytoplasm that is contiguous with one or more myofibers. As myofiber branching is thought to represent active/ incomplete fusion of myotubes and/or myofibers to each other ${ }^{41-44}$ the number of branched myofibers after muscle injury were quantified to gain insight into the extent to which 

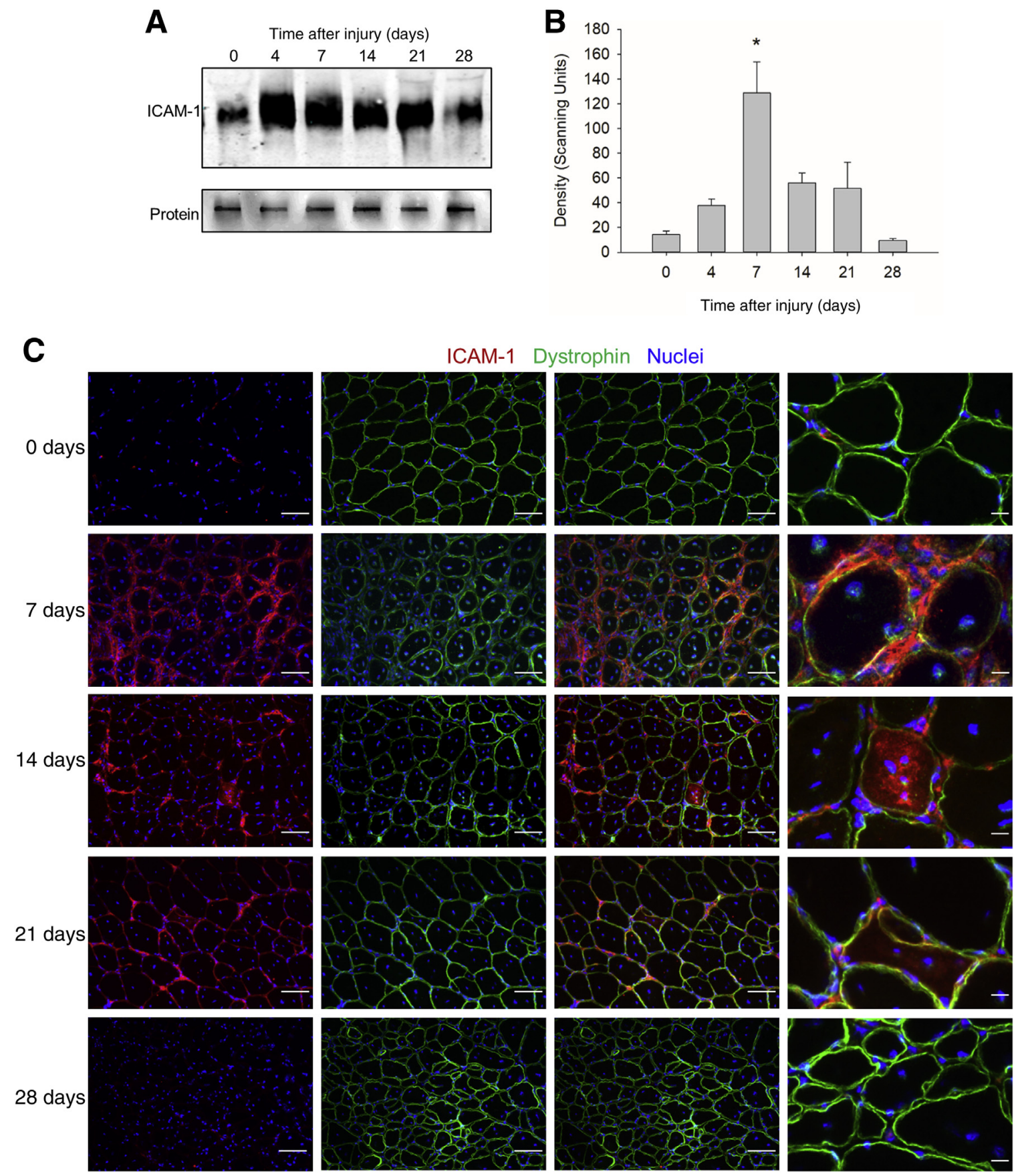

Figure 1 Elevated intercellular adhesion molecule-1 (ICAM-1) expression and localization after muscle injury. A: Western blot of ICAM-1 (110 kDa) in muscles of wild-type mice at $0,7,14,21$, and 28 days after injury (15 $\mu \mathrm{g}$ per lane). To serve as a control for sample loading, samples were loaded into a slot blot (15 $\mu \mathrm{g}$ per slot) and stained with Krypton fluorescent protein stain. B: Quantitative analysis of the relative abundance of ICAM-1 C: Images of ICAM-1 (red), dystrophin (green), and nuclei (blue) in muscle sections of wild-type mice at $0,7,14,21$, and 28 days after injury. Myofiber expression of ICAM-1 is indicated by the colocalization of ICAM-1 and dystrophin (yellow), as well as the detection of ICAM-1 in the sarcoplasm. The last column shows digital zoom images of the same field of view shown in the first three columns. Data are given as means \pm SEM (B). $n=6$ to 8 muscles per group (B). ${ }^{*} P<0.05$ versus all other time points. Scale bars: $10 \mu \mathrm{m}$ (C, right column); $50 \mu \mathrm{m}$ (C, left and middle columns).

ICAM-1 influences myotube/myofiber fusion (Figure 4). At 14 and 28 days after injury, the percentage of myofibers that exhibited at least one branched morphology (ie, bridged, bifurcated, or trifurcated end, or bifurcated side) (Figure 4A) was twofold lower for wild-type compared with $\operatorname{Icam} 1^{-1-}$ mice (Figure 4B) (interaction effect; $P<0.01$ ). Approximately $90 \%$ of the branched myofibers had a bridged morphology, and the frequency (number/myofiber) of such a morphology was increased for Icaml ${ }^{-1-}$ compared with wild-type mice (data not reported). All branched myofibers 
A

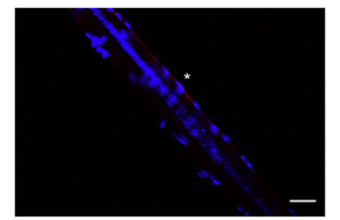

ICAM-1 Dystrophin Nuclei

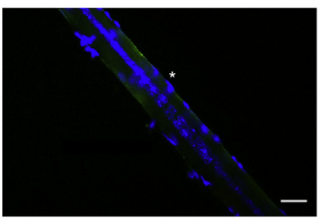

C
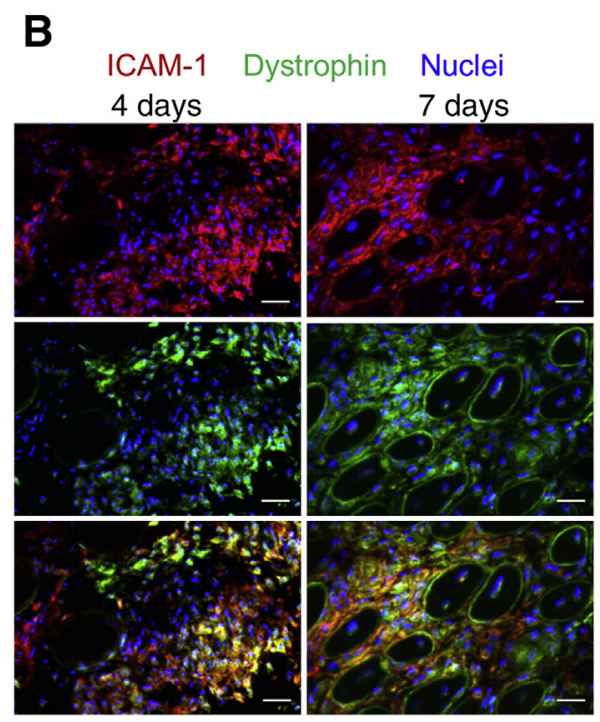

Nuclei 7 days

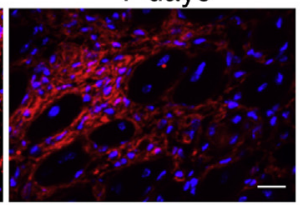

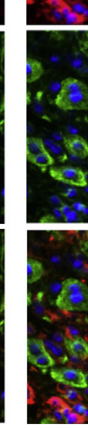
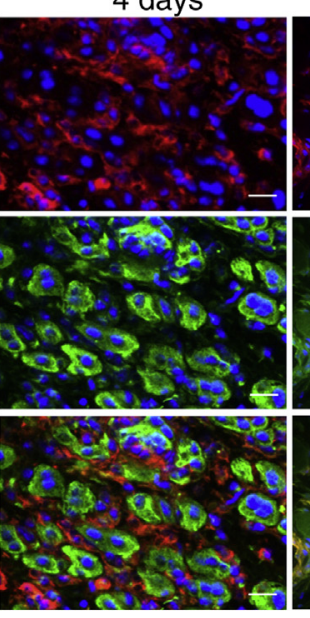
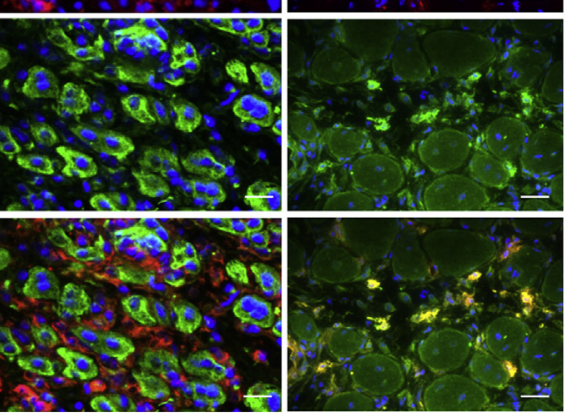

ICAM-1 Desmin Nuclei

7 days

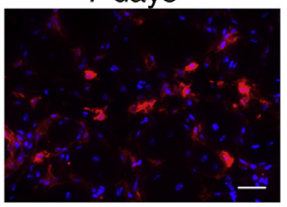

D

$\mathbf{E}$

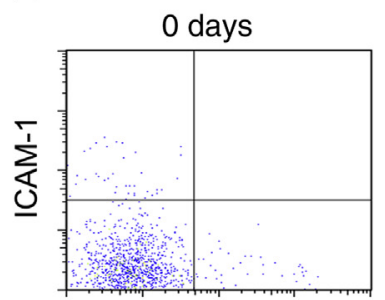

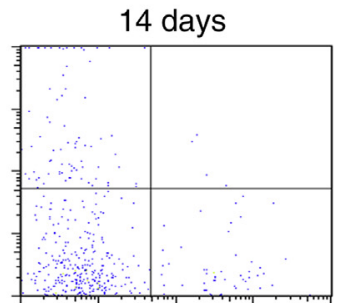

Integrin- $\alpha 7^{+}$
ICAM-1 GFP Nuclei
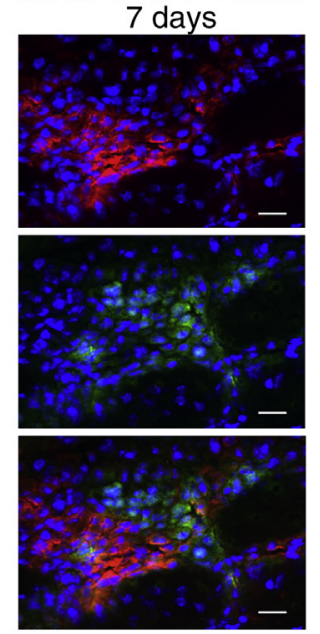

$\mathbf{F}$

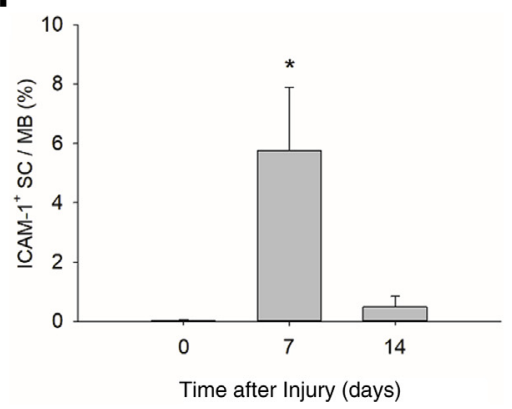

G

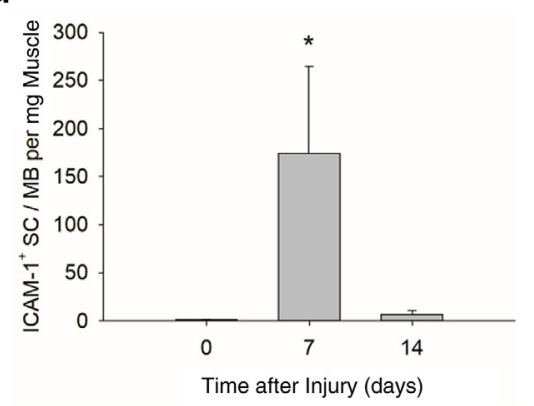

Figure 2 Intercellular adhesion molecule-1 (ICAM-1) expression by satellite cells/myoblasts and myofibers. A: Images of ICAM-1 (red), dystrophin (green), and nuclei (blue) in an isolated myofiber obtained from a wild-type mouse 7 days after injury. Asterisks indicate a presumptive satellite cell expressing ICAM1. B and C: Images of ICAM-1 (red), dystrophin or desmin (green), and nuclei (blue) in muscle sections of wild-type mice at 4 and 7 days after injury. Satellite cell/myoblast expression of ICAM-1 is indicated by the colocalization of ICAM-1 and dystrophin or desmin (yellow) in mononuclear cells. D: Images of ICAM-1

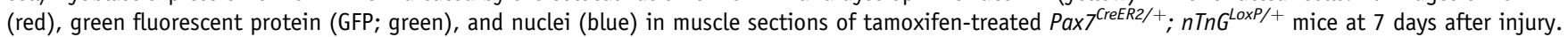
Satellite cell/myoblast expression of ICAM-1 is indicated by the colocalization of ICAM-1 and GFP (yellow) in mononuclear cells. E: Lin cells are defined as cells negative for CD45/CD11b/CD31/Sca-1. Flow cytometric analysis of ICAM-1 expression by Lin ${ }^{-}$integrin $\alpha 7^{+}$cells isolated from muscles of wild-type mice at 0 , 7 , and 14 days after injury. Thresholds for cells treated with the ICAM-1-allophycocyanin (APC) and integrin $\alpha 7-$ Alexa Fluor 488 antibodies were set on the basis of the fluorescent intensity of cells treated with an isotype control antibody. F: Percentage of satellite cells/myoblasts (SCs/MBs; Lin ${ }^{-}$integrin $\alpha 7^{+}$cells) that expressed ICAM-1 at 0,7 , and 14 days after injury. G: Number of ICAM- $1^{+}$SCs/MBs (Lin ${ }^{-}$integrin $\alpha 7^{+}$ICAM- $1^{+}$cells) per mg of muscle. Data are given as means $\pm \operatorname{SEM}(\mathbf{F}$ and $\mathbf{G}) . n=3$ mice per group $(\mathbf{F}$ and $\mathbf{G}) .{ }^{*} P<0.05$ versus all other time points. Scale bars: $50 \mu \mathrm{m}(\mathbf{A}) ; 20 \mu \mathrm{m}(\mathbf{B}-\mathbf{D})$. 


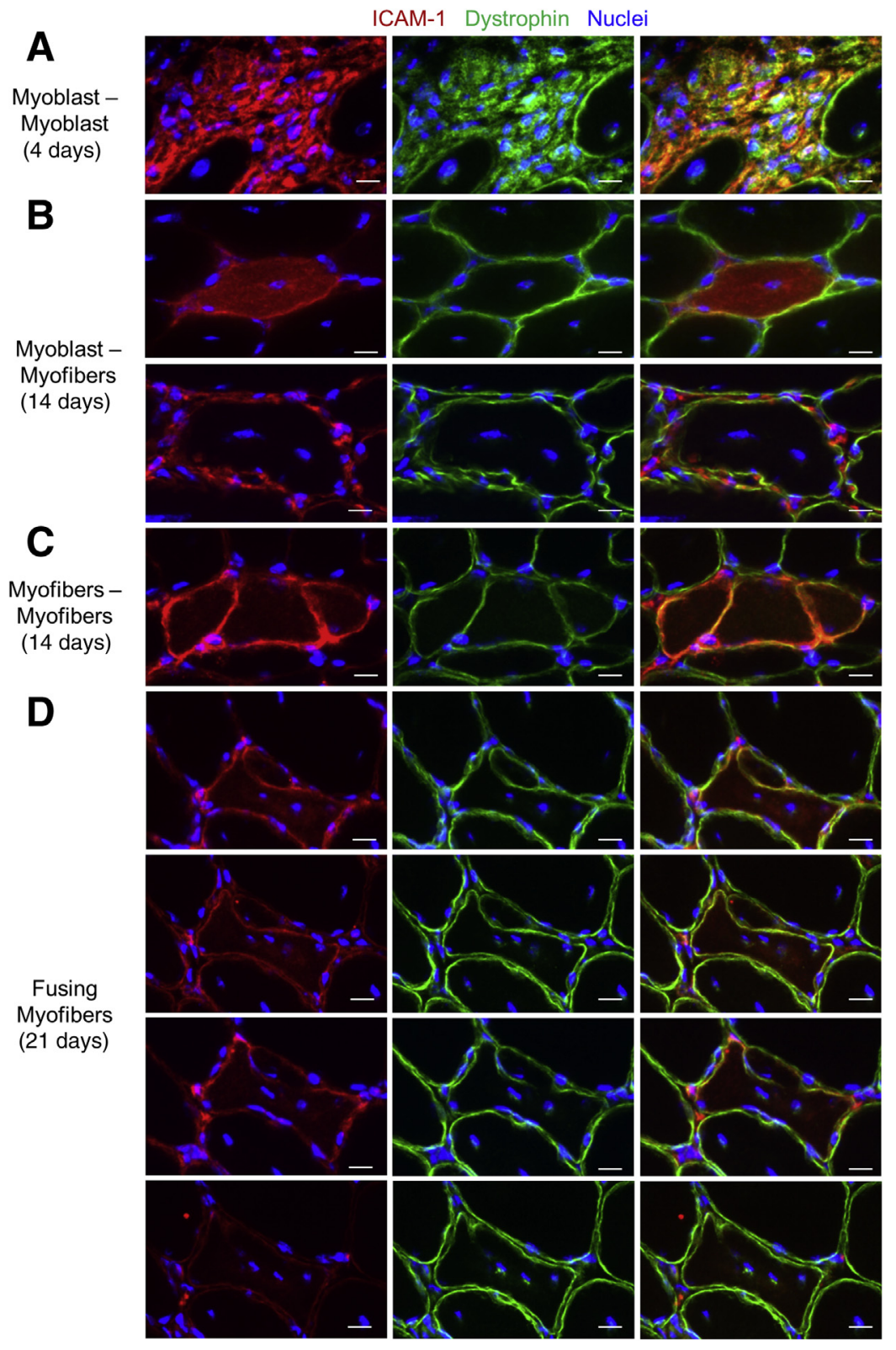

Figure 3 Intercellular adhesion molecule-1 (ICAM1)ICAM-1 interactions between opposing myogenic cells after muscle injury. Images of ICAM-1 (red), dystrophin (green), and nuclei (blue) in muscle sections of wild-type mice after injury. ICAM-1-ICAM-1 interactions are apparent between presumptive satellite cells/myoblasts (dystrophin ${ }^{+}$mononuclear cells; A), satellite cells/myoblasts and myofibers (B), and between myofibers (C), including those that appeared to be in the process of fusing together (D). Scale bars $=10 \mu \mathrm{m}(\mathbf{A}-\mathbf{D})$.

had centrally located nuclei and, thus, were regenerating myofibers. As the absence of ICAM-1 resulted in a greater number of branched myofibers after injury, ICAM-1 appears to facilitate fusion of myofibers after muscle injury. This interpretation is consistent with in vitro findings that ICAM-1 augments the parallel alignment of myotubes and their subsequent fusion to each other. ${ }^{21}$

\section{ICAM-1 Contributes to the Restoration of Muscle Structure after Injury}

The extent to which ICAM-1 facilitates the restoration of muscle structure after injury was determined by quantifying regenerating myofibers and myofiber cross- sectional area in muscle sections stained with wheat germ agglutinin (Figure 5A). In control muscles, the percentage of regenerating myofibers (wild type $=2.4 \% \pm 1.3 \%$; Icam $1^{-1-}=2.8 \% \pm 1.2 \% ; n=8$ muscles per genotype) and myofiber cross-sectional area (wild

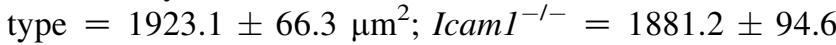
$\mu \mathrm{m}^{2} ; n=8$ muscles per genotype) were similar between the genotypes.

ICAM-1 contributed to regenerative processes after injury as the percentage of regenerating myofibers was $18 \%$ to $30 \%$ higher for wild-type compared with $\operatorname{Icam1}^{-1-}$ mice at 14, 21, and 28 days after injury (interaction; $P<0.05$ ) (Figure 5B). ICAM-1 also contributed to hypertrophic processes after injury, as indicated by differences between 

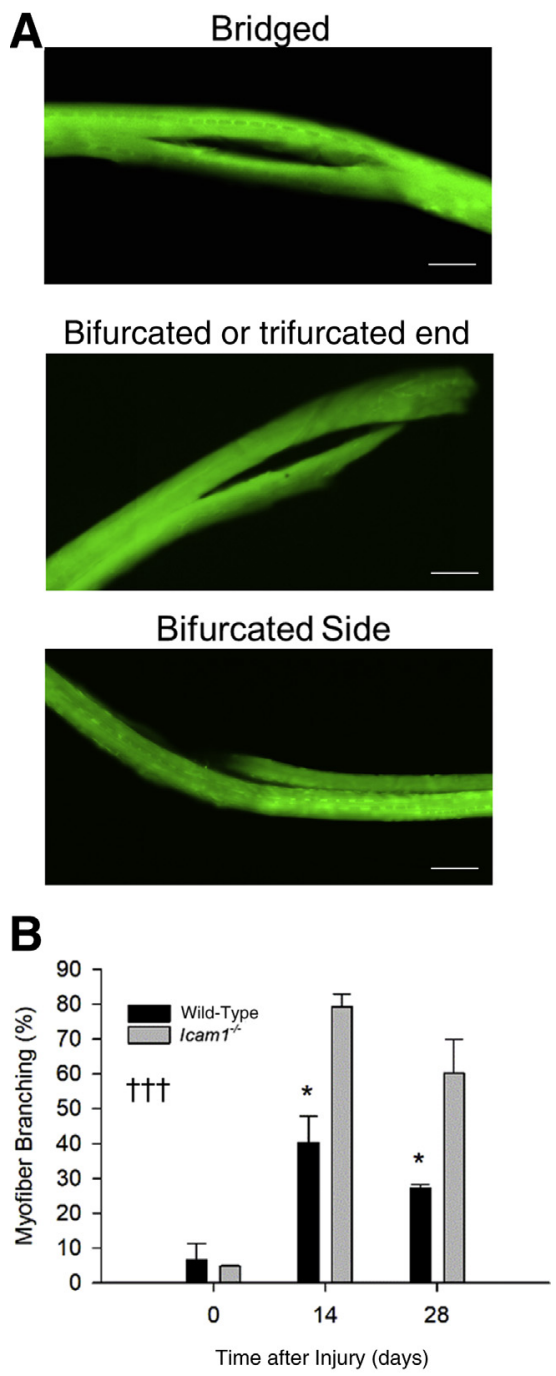

Figure 4 Increased myofiber branching in $\mathrm{Icam}^{-/-}$mice after injury. A: The z-plane images of branched myofibers. Myofibers that had a fissure that resembled a bridge or had a bifurcation on their side, or at their end, were scored as branched. Myofibers are delineated via formaldehydeinduced fluorescence (green). B: Percentage of myofibers within a muscle that were branched. Data are given as means \pm SEM (B). $n=3$ muscles per group (B). ${ }^{*} P<0.05$ versus Icam $1^{-/-}$at indicated time point (interaction); ${ }^{\dagger \dagger} P<0.001$ wild-type versus Icam $1^{-/-}$(main effect for genotype). Scale bars $=50 \mu \mathrm{m}(\mathbf{A})$.

the genotypes in myofiber cross-section area. Specifically, the cross-sectional area of myofibers after muscle injury was $23 \%$ to $48 \%$ higher for wild-type compared with Icaml $^{-1-}$ mice (main effect for genotype; $P<0.001$ ) (Figure 5C). Frequency distribution analysis of myofiber size revealed a clear difference between the genotypes at a bin of $1000 \mu \mathrm{m}^{2}$ (Figure 5D). Indeed, the percentage of small myofibers (200 to $1000 \mathrm{\mu m}^{2}$ ) within a muscle was approximately $50 \%$ higher in Icaml ${ }^{-1-}$ compared with wild-type mice at 7 to 28 days after injury (main effect for genotype; $P<0.001$ ) (Figure 5E). Correspondingly, the percentage of myofibers $>1000 \mu \mathrm{m}^{2}$ was lower for Icaml ${ }^{-l-}$ compared with wildtype mice after muscle injury (main effect for genotype; $P<0.001$ ) (Figure 5F).
ICAM-1 Does Not Influence the Restoration of Muscle Function after Injury

The peak torque of tibialis anterior muscles was quantified to determine the extent to which ICAM-1 influences the restoration of muscle function after muscle injury. Peak torque was similar in control muscles of wild-type $(1.01 \pm 0.07 \mathrm{~N} \cdot \mathrm{mm})$ and Icaml $^{-1-}(1.12 \pm 0.09$ $\mathrm{N} \cdot \mathrm{mm})$ mice. Peak torque was also similar between the genotypes at 7 to 28 days after injury (Figure 6A), although there was a trend toward a lower peak torque for wild-type compared with Icam $1^{-1-}$ mice after injury (main effect of genotype; $P=0.063$ ). However, this trend was negated when the data were expressed as a deficit relative to control values (Figure 6B). These findings indicate that ICAM-1 does not influence the restoration of muscle function after injury.

\section{ICAM-1 Contributes to Protein Synthesis after Injury}

Protein synthesis was measured via puromycin incorporation into proteins ${ }^{34,35}$ to identify a mechanism through which ICAM-1 facilitates the restoration of muscle structure after injury. Western blot detection of puromycin demonstrated that rates of protein synthesis was 12-fold and twofold higher in wild-type compared with $\mathrm{Icaml}^{-1-}$ mice at 7 and 14 days after injury, respectively (Figure 7, A-C) (interaction effect; $P<0.001$ ). To include proteins that were not resolved in $10 \%$ SDS-PAGE gels (ie, $>250$ and $<25$ $\mathrm{kDa}$ ) and to eliminate limitations of electrophoretic transfer of proteins to membranes, as well as imperfections in gels (eg, curved lane) and transfer membranes (eg, air bubbles), puromycin incorporation into proteins using a slot blot approach was also quantified (Figure 7D). This approach revealed that rates of protein synthesis were ninefold, threefold, and fourfold higher in wild-type compared with Icam $1^{-1-}$ mice at 7,14 , and 21 days after injury, respectively (Figure 7E) (interaction effect; $P<0.001$ ). Immunolabeling of muscle sections also revealed greater protein synthesis within myofibers of wild-type compared with ICAM-1 mice at 7 and 14 days after injury (Figure 7F). Taken together, these findings demonstrate that ICAM1 -mediated regenerative and hypertrophic processes after muscle injury are accompanied by greater rates of protein synthesis.

\section{ICAM-1 Alters the Number of Nuclei within Myofibers} after Injury

Fusion of satellite cells/myoblasts and myotubes to myofibers increases myonuclear number ${ }^{16,50}$ and, by conjecture, the capacity of myofibers to transcribe DNA and synthesize proteins. Thus, the number of nuclei within isolated single myofibers were quantified to gain insight into the extent to which ICAM-1 facilitates protein synthesis and myofiber 

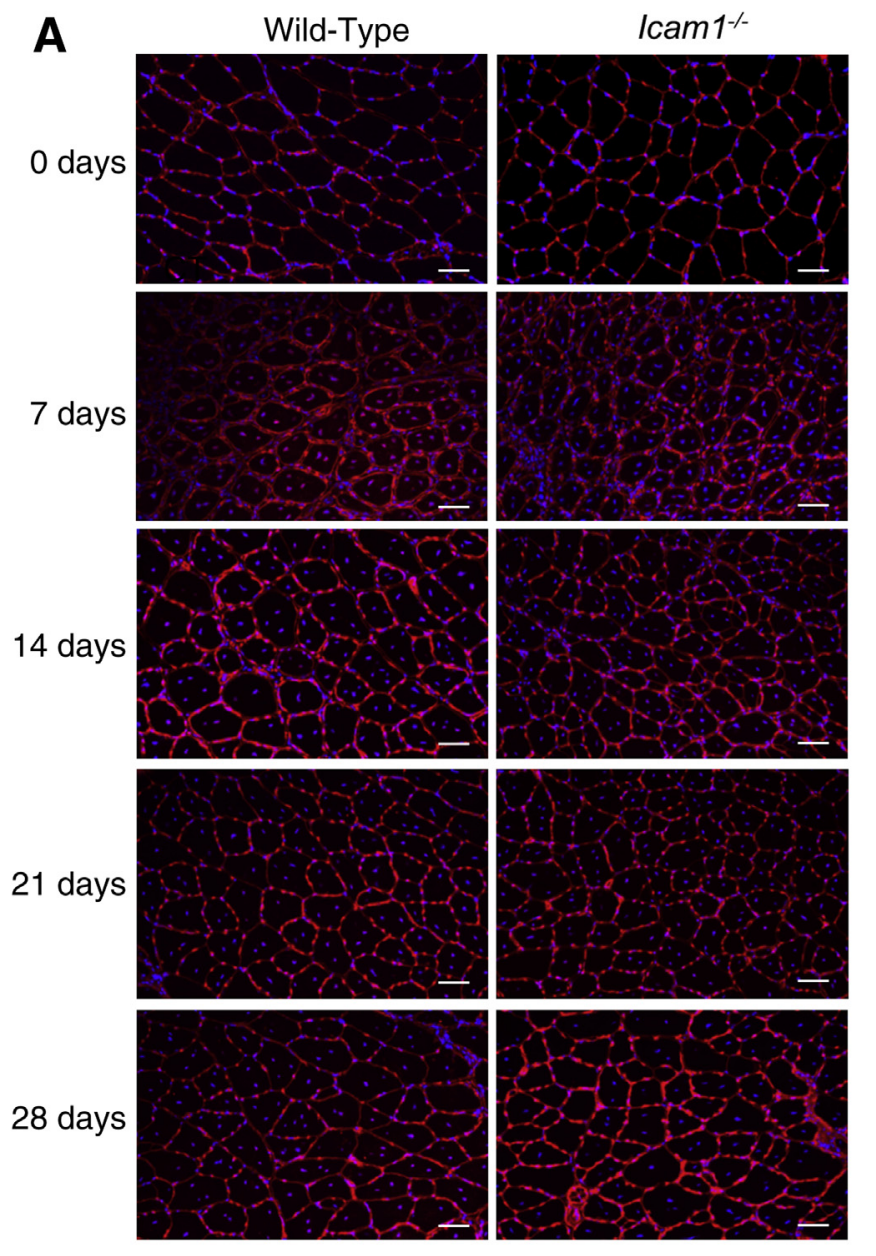

B
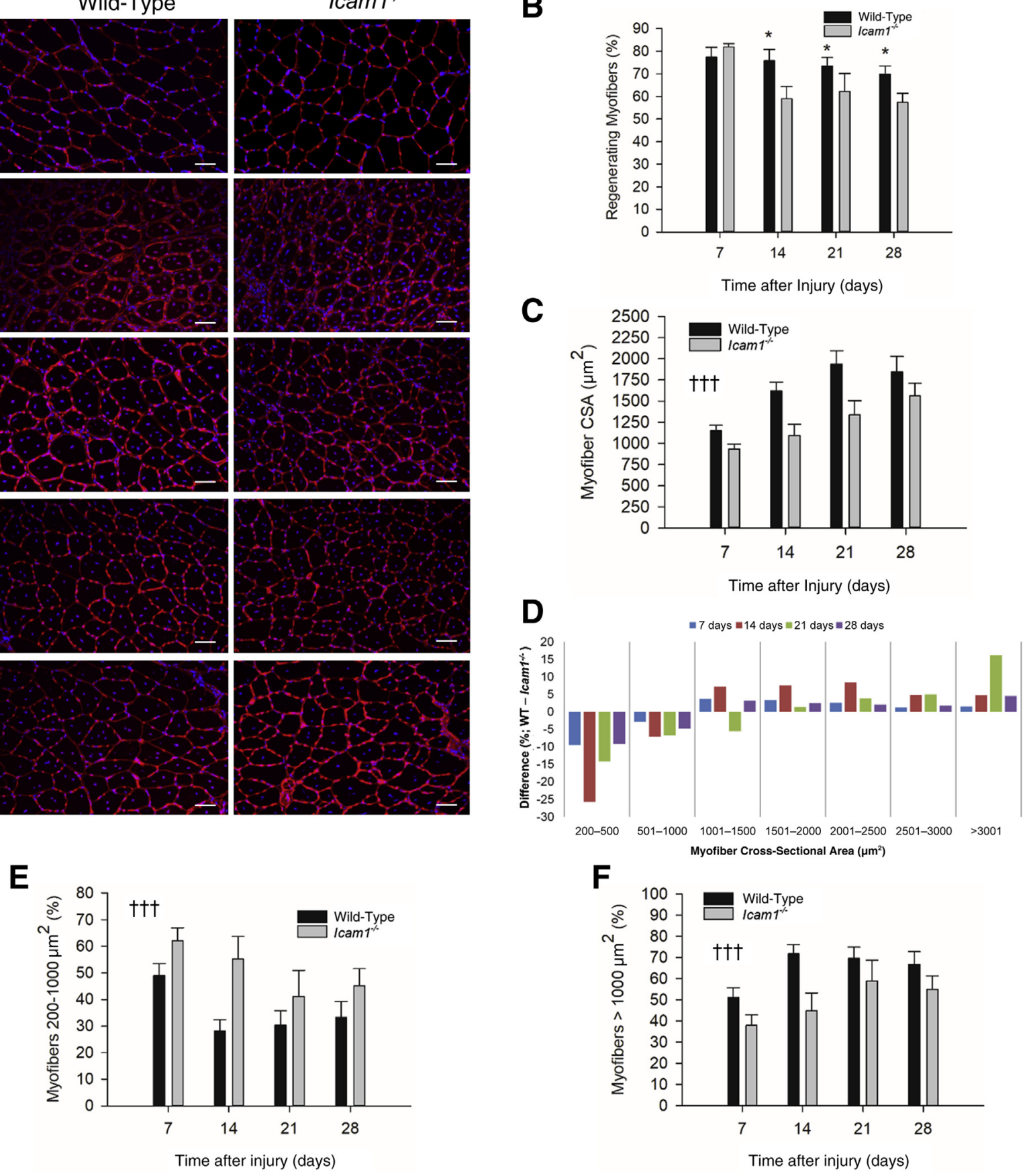

Figure 5 Intercellular adhesion molecule-1 (ICAM-1) contributes to the restoration of muscle structure. A: Images of wheat germ agglutinin (red) and nuclei (blue) in muscle sections of wild-type (WT) and Icam $1^{-1-}$ mice at $0,7,14,21$, and 28 days after injury. Quantitative analysis of myofibers at 7, 14, 21 , and 28 days after injury was performed on 556 myofibers per muscle (SD $= \pm 222$ myofibers) and 4437 myofibers per group (SD $= \pm 863$ myofibers). The total number of myofibers analyzed ( 0 to 28 days after injury) is 20,065 and 24,301 for wild-type and Icam1 ${ }^{-1-}$ mice, respectively. B: Percentage regenerating myofibers. C: Myofiber cross-sectional area $\left(\mu \mathrm{m}^{2}\right)$. D: Frequency distribution plot of myofiber cross-sectional area (CSA). The percentage of myofibers within bins was calculated for wild-type and Icam $1^{-1-}$ mice at each time point, and the absolute difference between the genotypes is plotted for ease of interpretation. E: Percentage of myofibers that had a cross-sectional area of 200 to $1000 \mu \mathrm{m}^{2}$. F: Percentage of myofibers that had a cross-sectional area that was greater than $1000 \mu \mathrm{m}^{2}$. Data are given as means \pm SEM $(\mathbf{B}-\mathbf{F}) . n=8$ muscles per group $(\mathbf{B}-\mathbf{F}) .{ }^{*} P<0.05,{ }^{* *} P<0.001$ versus Icam $1^{-/-}$at indicated time point; ${ }^{\dagger \dagger} P<0.001$ wild-type versus Icam $1^{-1-}$ (main effect for genotype). Scale bars $=50 \mu \mathrm{m}(\mathbf{A})$.

hypertrophy through a mechanism involving myonuclear accretion.

At 14 and 28 days after injury, nuclei within myofibers were closely aligned in a linear array, which has been termed nuclear chains ${ }^{45}$ (Figure 8, A and C). Nuclear chains were often running in parallel tracks, and some chains diverged/converged to produce a Y-shaped appearance. These organizational patterns of nuclei were frequently, but 

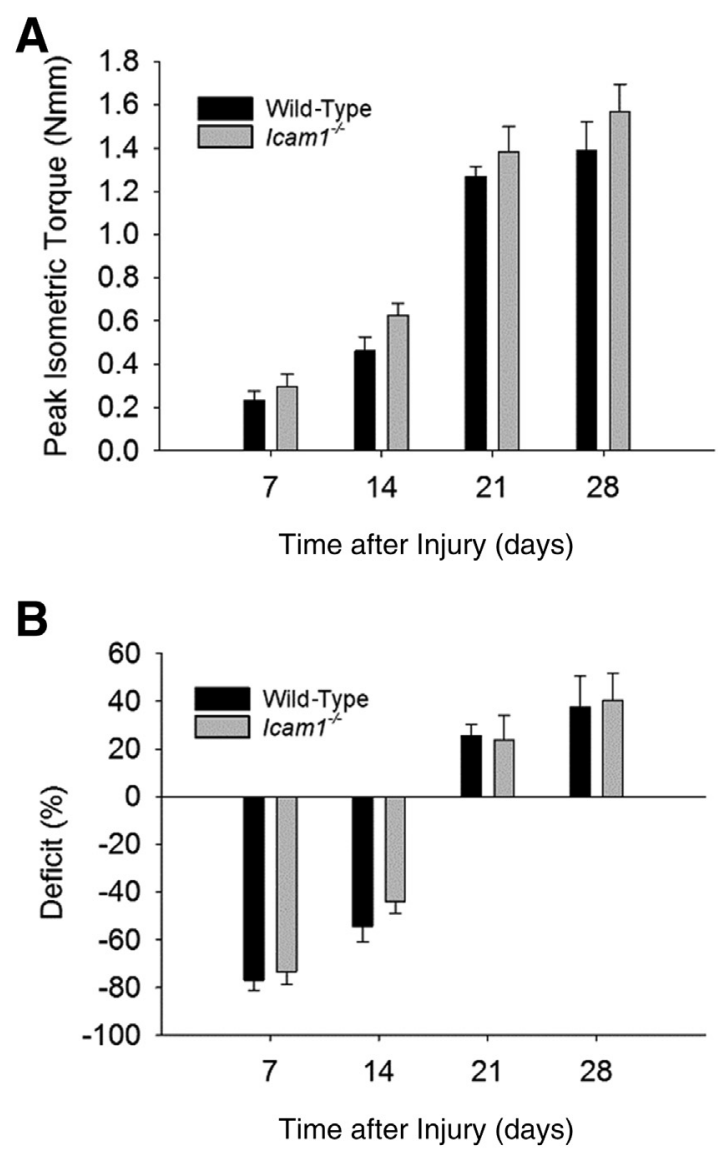

Figure 6 Intercellular adhesion molecule-1 (ICAM-1) does not contribute to the restoration of muscle function after injury. A: Peak torque of tibialis anterior muscles after injury. B: Peak torque of tibialis anterior muscles expressed as a deficit relative to control values for each genotype. Data are given as means \pm SEM (A and $\mathbf{B}$ ). $n=6$ to 9 muscles per group (A and $\mathbf{B}$ ).

not exclusively, found in segments of myofibers that exhibited a bridged or bifurcated side morphology. Clusters of nuclei, which was defined as a nonlinear grouping of three or more nuclei, were also found within myofibers after muscle injury (Figure 8, B and C). Clusters of nuclei were not restricted to specific regions of myofibers (eg, ends or middle). No nuclear chains and few clusters of nuclei were observed within myofibers from control mice.

Given the close proximity of nuclei within nuclear chains and clusters, as well as changes in nuclear morphology, it was difficult to count nuclei in z-projections of myofibers isolated after muscle injury, even after image deconvolution, image segmentation, and the use of an algorithm for counting nuclei within clusters. To improve accuracy, nuclei in single z-plane images were quantified in which most nuclei were in focus and near the center of the myofiber. This approach, coupled with image segmentation and the use of the cluster algorithm, greatly improved the accuracy of counting nuclei within myofibers after muscle injury. Thus, data reported herein were obtained from single z-plane images of myofibers near their centroid. Nuclei not visible in selected z-plane images were not counted, and thus, the reported number of nuclei within single myofibers should be interpreted to be representative of a relative large area of a myofiber (63\% of myofiber length; approximately $220 \mathrm{~mm}^{2}$ ), as opposed to the number of nuclei per unit myofiber volume.

The number of nuclei standardized to myofiber length (nuclei/100 $\mu \mathrm{m}$ ) and area (nuclei $/ \mathrm{mm}^{2}$ ) was similar in control muscles of wild-type and Icam1 ${ }^{-1-}$ mice (Figure 8, $\mathrm{D}-\mathrm{F}$ ), as well as to values reported in the literature for tibialis anterior muscles of wild-type mice. ${ }^{40}$ For wild-type mice, the number of nuclei (nuclei/100 $\mu \mathrm{m}$ and nuclei/ $\mathrm{mm}^{2}$ ) at 14 and 28 days after injury increased above control levels by twofold to threefold. Unexpectedly, the number of nuclei (nuclei/100 $\mu \mathrm{m}$ and nuclei $/ \mathrm{mm}^{2}$ ) was $30 \%$ to $35 \%$ lower after muscle injury for myofibers of wild-type compared with Icam1 ${ }^{-1-}$ mice (main effect of genotype $P<0.05$; interaction effect $P<0.05$ ). Frequency distribution analysis of myofibers after injury revealed a clear difference between the genotypes at a bin of 20 nuclei/100 $\mu \mathrm{m}$ (Figure $8 \mathrm{E}$ ). Indeed, $45 \%$ of the wild-type myofibers at 14 and 28 days after injury had $<20$ nuclei/100 $\mu \mathrm{m}$, whereas $19 \%$ of the Icam1 ${ }^{-I-}$ myofibers had $<20$ nuclei/ $100 \mu \mathrm{m}$ at 14 and 28 days after injury.

These findings indicate that either ICAM-1 inhibits myonuclear accretion after muscle injury or a compensatory increase in myonuclear accretion occurred in the absence of ICAM-1. We favor the later interpretation as the interpretation that ICAM-1 inhibits myonuclear accretion after muscle injury is not congruent with prior research that demonstrated that ICAM-1 augments myonuclear accretion in cultured myotubes. ${ }^{21,22}$

\section{ICAM-1 Alters the Organization of Nuclei within Myofibers after Injury}

While counting nuclei within myofibers, striking differences were observed between the genotypes in the length of nuclear chains and number of clustered nuclei. The length of nuclear chains varied widely within a single myofiber of wild-type mice after injury, with the longest chains spanning $40 \%$ to $95 \%$ of myofiber length. More important, the average length of nuclear chains within a myofiber at 14 and 28 days after injury was twofold longer for wild-type compared with Icaml $^{-1-}$ mice (main effect for genotype; $P<0.001$ ) (Figure $8 \mathrm{G}$ ). Interestingly, nuclear chains were approximately $20 \%$ longer in both genotypes at 28 compared with 14 days after injury, suggesting that chain elongation accompanies muscle regeneration. Qualitative observations indicated that myofibers of wild-type mice also had more parallel and Y-shaped tracks of nuclei compared with myofibers of Icaml ${ }^{-1-}$ mice. The percentage of nuclei found in clusters (main effect of genotype; $P<0.05$ ) (Figure $8 \mathrm{H}$ ) as well as the number of clusters (Figure 8, I and J) (clusters $/ 100 \mu \mathrm{m}$ : main effect of genotype $P<0.05$; clusters $/ \mathrm{mm}^{2}$ : interaction effect $\left.P<0.05\right)$ were two to 
A

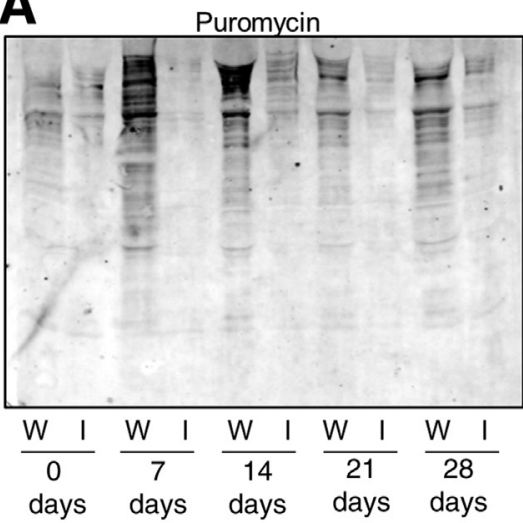

B

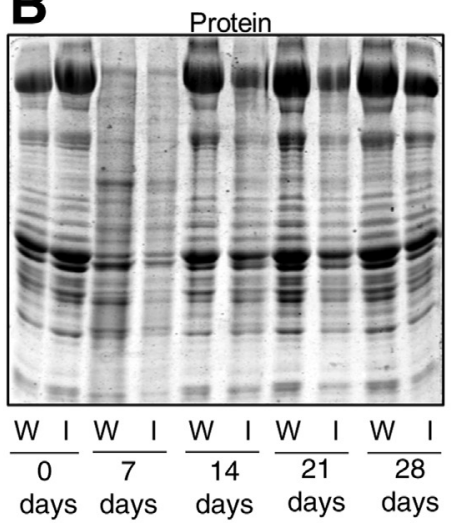

C

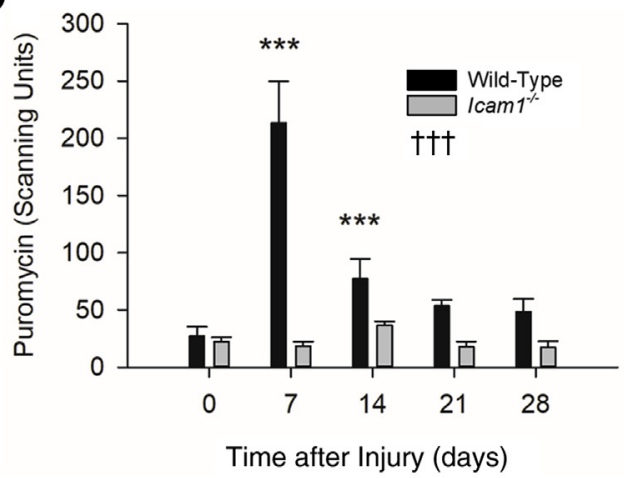

D

Time after Injury (days)

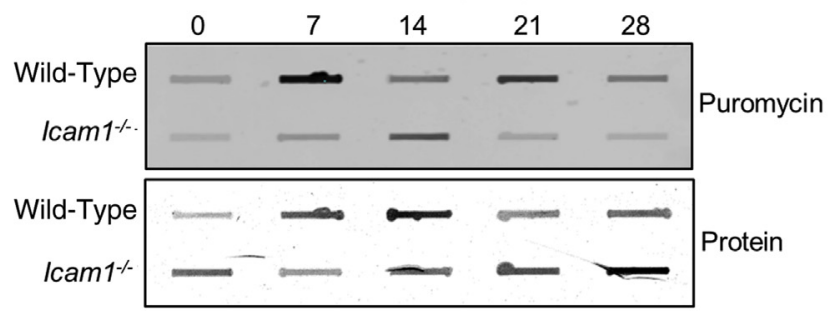

E

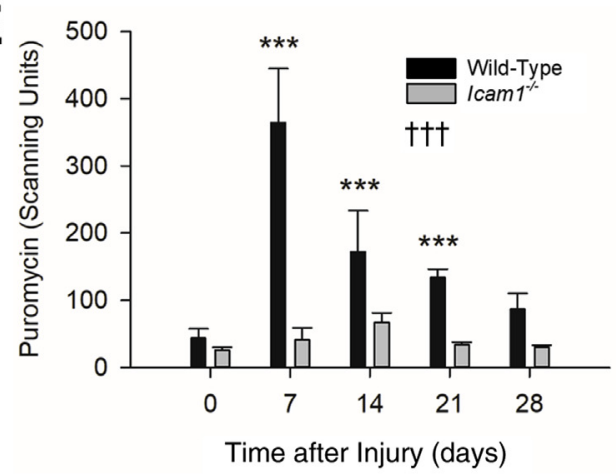

F
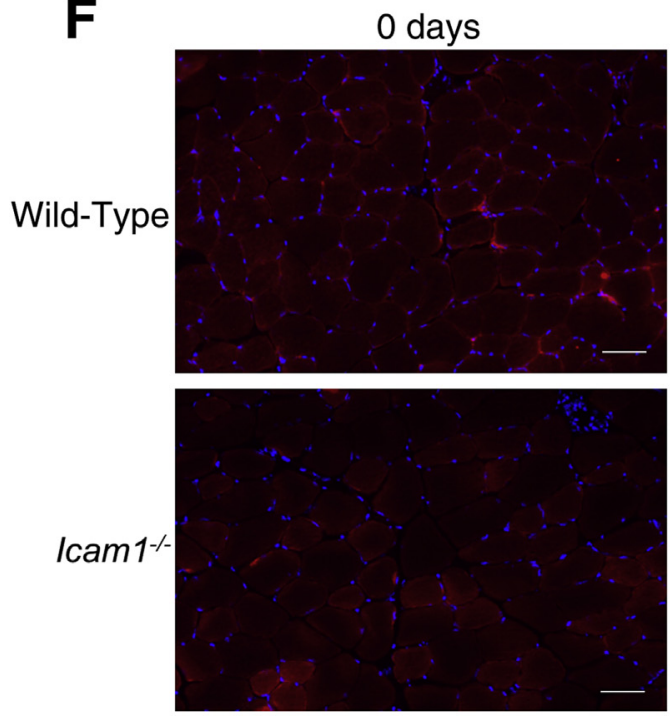

7 days
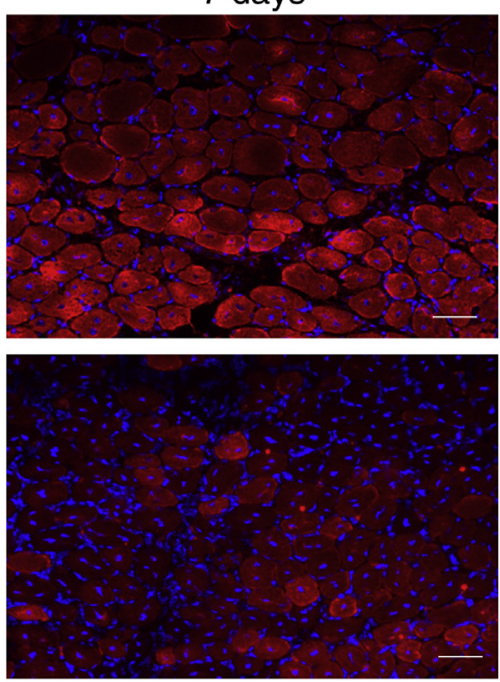

14 days
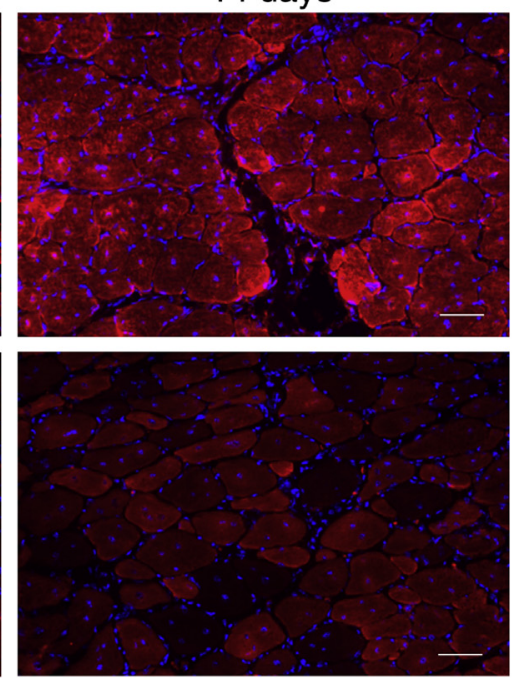

Figure 7 Intercellular adhesion molecule-1 (ICAM-1) contributes to increased rates in protein synthesis after muscle injury. A: Western blot detection of puromycin within muscles of wild-type (W) and Icam1 ${ }^{-1-}$ (I) mice after muscle injury (50 $\mu \mathrm{g}$ protein/lane). B: Coomassie Blue stained $10 \%$ SDS-PAGE gel (control for sample loading) containing muscle homogenates from W and I mice (50 $\mu \mathrm{g}$ protein/lane). C: Quantitative analysis of Western blot detection of puromycin incorporation into proteins. D: Slot blot detection of puromycin and total protein (Ponceau stain) within muscle homogenates of W and I mice $(15 \mu \mathrm{g} / \mathrm{slot})$. E: Quantitative analysis of puromycin using the slot blot approach. F: Images of puromycin (red) and nuclei (blue) in muscle sections of wild-type and Icam1 ${ }^{-1-}$ mice at 0,7 , and 14 days after injury. Puromycin incorporation into proteins of myofibers is indicated by the detection of puromycin in the sarcoplasm. Data are given as means \pm SEM $(\mathbf{C}$ and $\mathbf{E}) . n=4$ muscles per group $(\mathbf{C}$ and $\mathbf{E}) .{ }^{* \star *} P<0.001$ versus Icam $1^{-1-}$ at indicated time point; ${ }_{\dagger \dagger \dagger} P<0.001$ wild-type versus Icam $1^{-/-}$(main effect for genotype). Scale bars $=50 \mu \mathrm{m}(\mathbf{F})$.

fourfold lower for wild-type compared with Icaml $^{-1-}$ mice at 14 and 28 days after injury. The number of nuclei per cluster in control myofibers was similar between the genotypes (wild type $=3.4 \pm 0.3 ;$ Icaml $^{-1-}=3.1 \pm 0.3$ ) and lower in wild-type compared with Icaml $^{-1-}$ mice at 14 (wild type $=4.9 \pm 0.3 ;$ Icaml $^{-1-}=6.3 \pm 0.2$ ) and 28 (wild type $=4.5 \pm 0.1 ;$ Icaml $^{-1-}=5.3 \pm 0.2$ ) days after injury (main effect of genotype; $P<0.05$ ). 
A
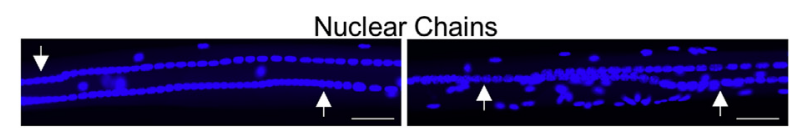

C

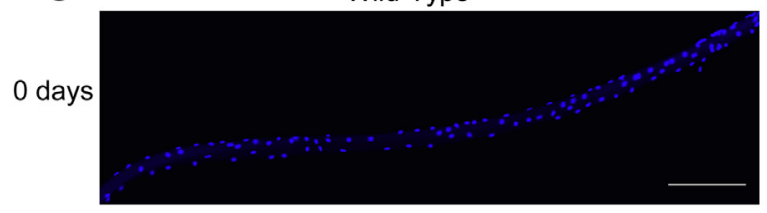

Wild-Type
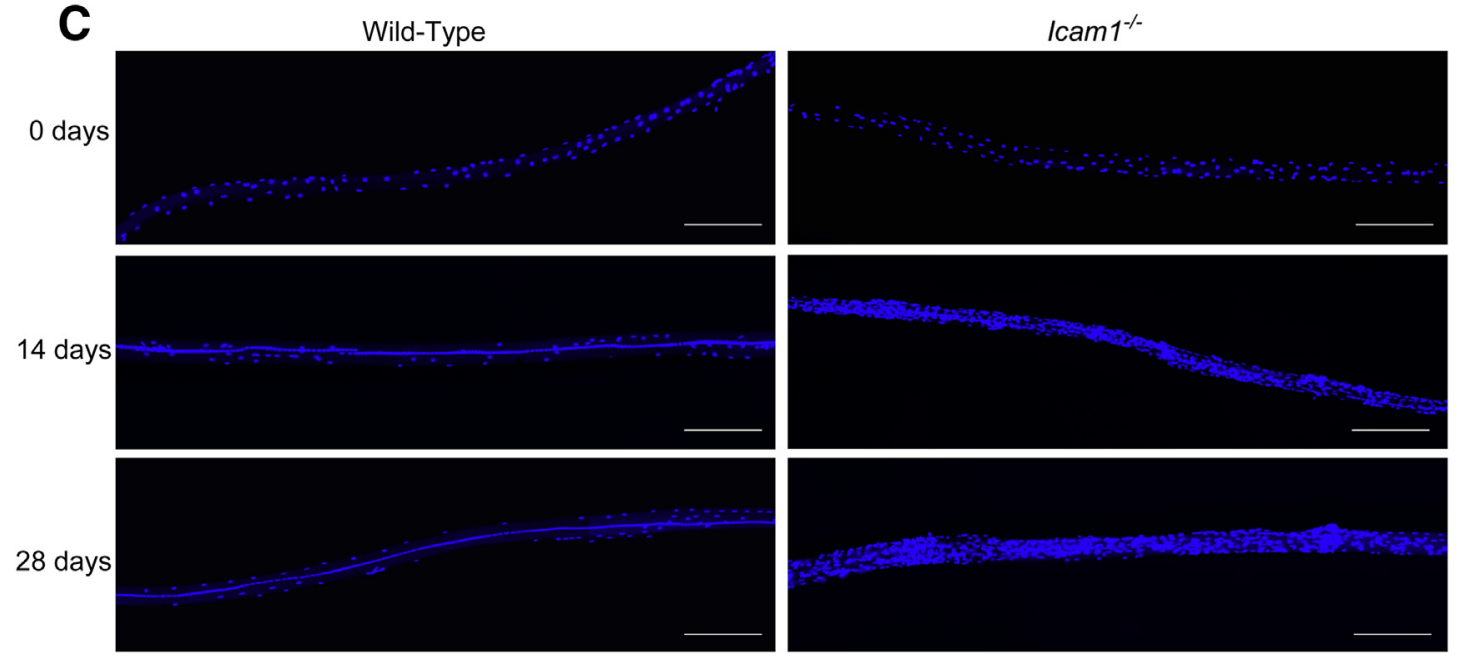

D

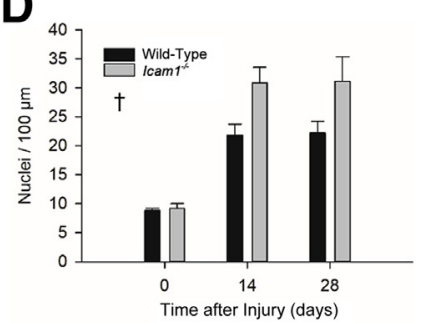

E

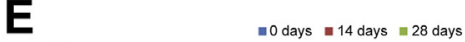

E $\quad 0$ days $\| 14$ days $\| 28$ days
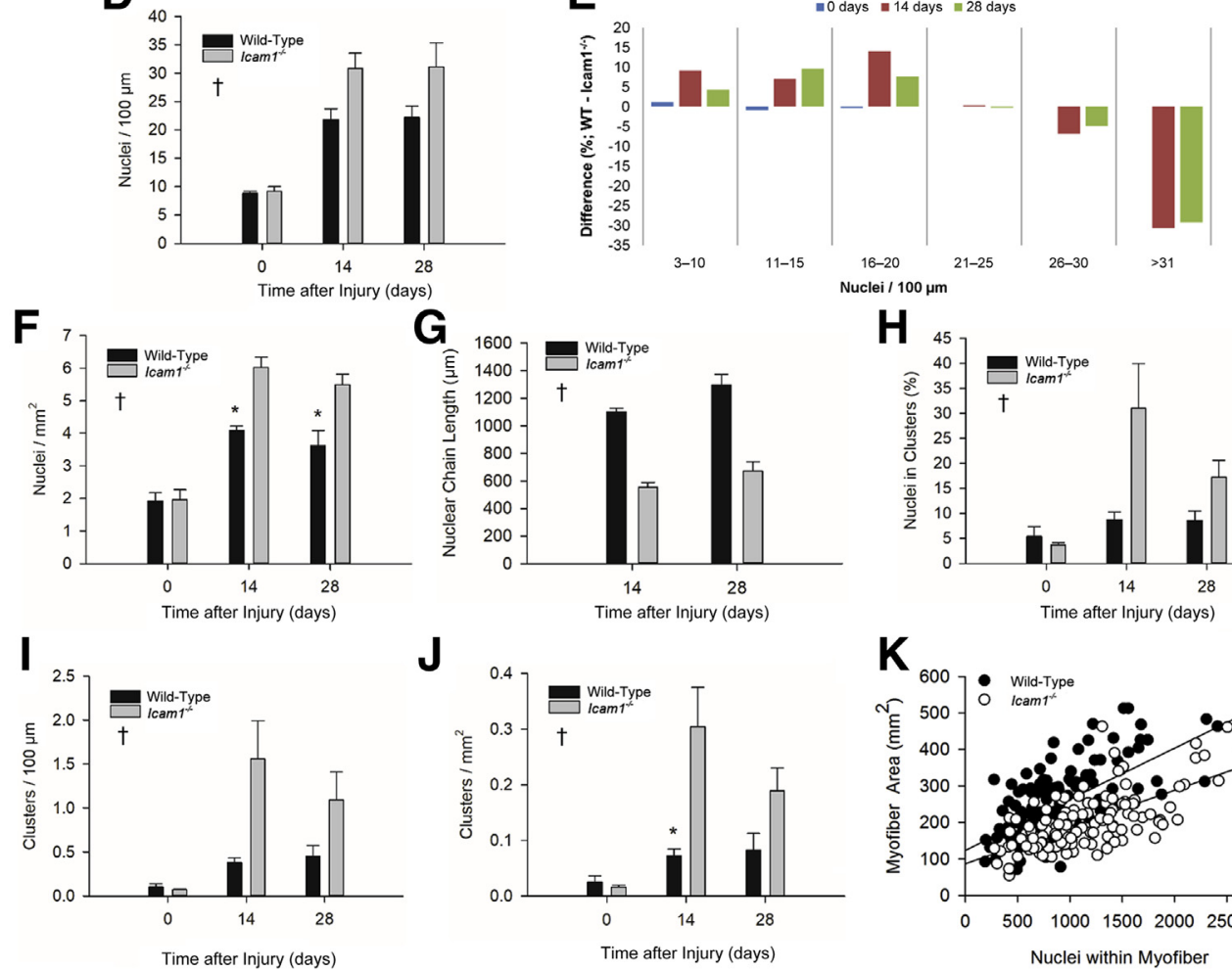

H

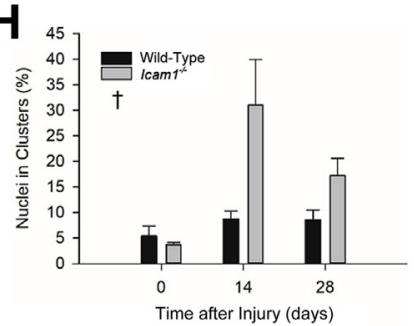

J

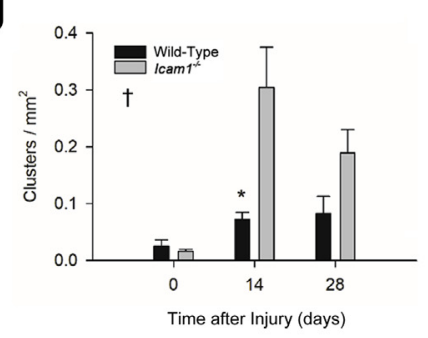

Figure 8 Intercellular adhesion molecule-1 (ICAM-1) influences nuclear number and organization in single myofibers. A and B: The z-plane images of nuclei (blue) within myofibers after muscle injury. Nuclei are found organized in long linear arrays called nuclear chains (arrows) or in clusters (arrowhead). C: The z-plane images of nuclei (blue) within myofibers of wild-type (WT) and Icam1 $1^{-1-}$ mice at 0,14 , and 28 days after injury. Quantitative analysis of nuclei within single myofibers was performed on 23 myofibers per muscle (18 to 26 myofibers per muscle) and 3 muscles per group (D-K). The total number of myofibers analyzed is 201 and 199 for wild-type and Icam $1^{-/-}$mice, respectively. D: Number of nuclei standardized to myofiber length (100 $\left.\mu \mathrm{m}\right)$. E: Frequency distribution plot of nuclei $/ 100 \mu \mathrm{m}$. The percentage of myofibers within bins was calculated for wild-type and Icam1 ${ }^{-1-}$ mice at each time point, and the absolute difference between the genotypes is plotted for ease of interpretation. F: Number of nuclei standardized to myofiber area $\left(\mathrm{mm}^{2}\right)$. G: Length of nuclear chains $(\mu \mathrm{m})$. H: Percentage of nuclei found in clusters. I: Number of clusters of nuclei standardized to myofiber length (clusters/100 $\mu \mathrm{m})$. J: Number of clusters of nuclei standardized to myofiber area (clusters $/ \mathrm{mm}^{2}$ ). K: Scatterplot and regression line (black lines) for the number of nuclei within a myofiber segment and its corresponding area $\left(\mathrm{mm}^{2}\right)$ for myofibers of wild-type [myofiber area $=(0.140 \times$ number of nuclei $)+123.3$ ] and Icam $1^{-/-}[\mathrm{myofiber}$ area $=(0.100 \times$ number of nuclei $)+86.6]$ mice at 14 and 28 days after injury. Data are given as means \pm SEM $(\mathbf{C})$. $n=135$ myofibers wild-type mice (K); $n=134$ myofibers Icam $1^{-/-}$mice (K). ${ }^{*} P<0.05$ wild-type versus Icam $1^{-/-}$at indicated time point (interaction effect); ${ }^{\dagger} P<0.05$ wild-type versus Icam $1^{-1-}$ (main effect for genotype). Scale bars: $50 \mu \mathrm{m}$ (A and B); $200 \mu \mathrm{m}$ (C). 
Taken together, these findings demonstrate a role of ICAM-1 in the organization of nuclei within myofibers during injury-induced muscle regeneration. The extent to which nuclear positioning/organization impacts the phenotype of myofibers after injury, however, is unknown.

\section{Relationship between Nuclei and Myofiber Size after Injury}

To explore the contribution of myonuclear accretion to the regulation of myofiber size after muscle injury, correlational analysis was performed using the number of nuclei within a myofiber segment and its corresponding area on a data set that included both the 14 and 28 days after injury time points (Figure $8 \mathrm{~K}$ ). Pearson product moment correlation coefficients were similar for wild-type $(r=0.67, P<0.001 ; n=135$ myofibers $)$ and Icaml $^{-l-}(r=0.65, P<0.001 ; n=134$ myofibers) mice. However, regression analysis between wildtype [myofiber area $=(0.140 \times$ number of nuclei $)+123.3$ ] and Icaml $^{-1-}$ [myofiber area $=(0.100 \times$ number of nuclei) +86.6$]$ mice (Figure $8 \mathrm{~K}$ ) indicated differences for slope $(P<0.05)$. Differences between the genotypes in the regression line slope indicate that the restoration of myofiber size after injury was more responsive to myonuclear accretion for wild-type compared with $\mathrm{Icaml}^{-1-}$ mice. Stated differently, a greater increase in the number of nuclei within myofibers of Icaml $1^{-1-}$ compared with wild-type mice was needed to achieve an equivalent increase in myofiber size.

\section{Discussion}

Adhesion of myogenic cells to each other is critically important in muscle regeneration as it triggers their fusion (ie, membrane union), resulting in myotube formation and an increased number of nuclei within myotubes and myofibers. ${ }^{16,50}$ Little, however, is known about membrane structures that regulate homotypic adhesion and fusion of myogenic cells, particularly after muscle injury. We report herein that muscle injury induced the expression of ICAM-1 by satellite cells/myoblasts and myofibers, and that their expression of ICAM-1 facilitated the restoration of muscle structure, but not function, after injury. Specifically, ICAM-1 contributed to injury-induced regulation of myofiber branching, regenerating myofiber number, myofiber size, and protein synthesis, as well as influenced the number and organization of nuclei within myofibers. Our findings, in conjunction with prior work, ${ }^{13,21,22}$ support a paradigm in which ICAM-1 augments the adhesive and fusogenic properties of myogenic cells, which, in turn, facilitates regenerative and hypertrophic processes that restore muscle structure after injury.

ICAM-1 is a member of Ig superfamily of adhesion molecules that is not constitutively expressed by satellite cells and myofibers in control muscles and by cultured myoblasts and myotubes. ${ }^{13,20-27,29}$ Myofiber expression of ICAM-1 was first reported in patients with inflammatory myopathies, ${ }^{27-29}$ and subsequently reports showed myogenic cell expression of ICAM-1 in murine models of skeletal muscle hypertrophy ${ }^{13}$ and Duchenne muscular dystrophy. ${ }^{20}$ A novel finding of the present study was that muscle injury caused ICAM-1 to be expressed by a population of satellite cells/myoblasts and myofibers. As skeletal muscle injury, hypertrophy, and myopathies elicit a local inflammatory response, ${ }^{1-4,51}$ induced expression of ICAM-1 by myogenic cells appears to reflect the inflammatory milieu of skeletal muscle, as opposed to the extent to which skeletal muscle shows signs of regeneration, hypertrophy, or disease (ie, the phenotype of skeletal muscle). Previous investigators have reported that tumor necrosis factor- $\alpha$, interferon- $\gamma$, IL- 4 , and IL- $1 \beta$ induce ICAM- 1 expression by cultured myoblasts and/or myotubes, ${ }^{13,23-26}$ whereas transforming growth factor- $\beta 1$ and IL-10 inhibit cytokineinduced expression of ICAM-1 by cultured myoblasts. ${ }^{24}$ As these cytokines are elevated within regenerating muscle after injury, ${ }^{51-54}$ speculation is that multiple cytokines within skeletal muscle temporally regulate myogenic cell expression of ICAM-1 after muscle injury.

A major finding of the present study is that ICAM-1 contributed to the restoration of muscle structure after injury by regulating myofiber branching, myofiber size, and protein synthesis after injury. These findings are in agreement with prior work that demonstrated that ICAM-1 contributes to regeneration and hypertrophy after muscle overload ${ }^{13}$ and augments regenerative and hypertrophic processes of in vitro myogenesis. ${ }^{21,22}$ In the current study's in vitro model, forced expression of ICAM-1 by myogenic cells augmented their adhesion to each other through homophilic trans interactions, and their subsequent fusion through membrane and cytoskeletal dynamics, resulting from adhesion-induced ICAM-1 signaling. ${ }^{21,22}$ Such mechanisms may be operating in vivo after muscle injury, as ICAM- $1^{+}$ myogenic cells were found closely associated with each other and a greater number of branched myofibers was seen in $I$ cam $1^{-I-}$ mice compared with wild-type mice. These findings are consistent with the premise that ICAM-1 facilitates myofiber fusion after muscle injury. The contribution of ICAM-1 to the regulation of myofiber size and protein synthesis after muscle injury (present study) and overload $^{13}$ is also consistent with our in vitro findings that ICAM-1 signaling augmented myotube size and protein synthesis through adhesion-induced myotube-myotube fusion and Akt/p70 s6k signaling. ${ }^{21,22}$

A novel aspect of the present study is the quantification of the number and organization of nuclei within single myofibers after muscle injury. Normally, most nuclei within myofibers are nonrandomly dispersed and positioned in a peripheral location near the sarcolemma, with a small subset of nuclei forming clusters at the neuromuscular and myotendinous junctions. ${ }^{55-57}$ After muscle injury, nuclei of satellite cells/myoblasts take a central position within newly formed myotubes/myofibers after fusion, and align in linear arrays (ie, nuclear chains). ${ }^{16,42,53-55}$ Interestingly, nuclear chains were longer in myofibers of wild-type compared with 
Icam $1^{-1-}$ mice after injury. Nuclear chain length influences the probability of identifying myofibers in transverse sections as regenerating, as long nuclear chains increase the likelihood of detecting myofibers with central nucleation in any given transverse plane of muscle. Thus, the lower percentage of regenerating myofibers observed in $\mathrm{Icaml}^{-1-}$ compared with wild-type mice after injury could be attributable to the shorter nuclear chains observed in myofibers of Icaml $1^{-1-}$ mice, as opposed to indicating a role of ICAM-1 in de novo myofiber formation.

Myofibers formed after injury are thought to continue to add nuclei via satellite cell/myoblast and myotube/myofiber fusion until they become a normal sized myofiber with peripheral nuclei. ${ }^{16,50,56,57}$ The resulting myonuclear accretion increases the number of nuclei that are available for transcription, which, in theory, facilitates myofiber hypertrophy through mechanisms that govern protein synthesis and myofibrillogenesis. The statistical correlation between nuclei number and myofiber area $(r=0.67)$ and the temporal pattern of protein synthesis and myofiber hypertrophy observed in wild-type mice after injury are consistent with myonuclear accretion paradigm for myofiber hypertrophy after injury. However, direct evidence supporting such a paradigm is lacking. Furthermore, mechanisms that govern the position (eg, peripheral versus central), organization (eg, chains and clusters), and migration of nuclei within myofibers after injury remains to be determined, although proteins of the linker of nucleoskeleton and cytoskeleton complex and dynamic changes in the cytoskeleton are likely to be involved. ${ }^{56-58}$

Given the paradigm for myofiber hypertrophy after injury, it was hypothesized that the number of nuclei within myofibers after muscle injury would be higher for wild-type compared with Icaml ${ }^{-1-}$ mice. Contrary to our hypothesis, the number of nuclei standardized to myofiber length (nuclei/100 $\mu \mathrm{m}$ ) and area (nuclei $/ \mathrm{mm}^{2}$ ) was lower for wild-type compared with Icaml ${ }^{-1-}$ type mice after injury. Surprisingly, differences were observed between the genotypes in the organization of nuclei within myofibers (namely, the higher prevalence of clusters of nuclei within myofibers of Icaml ${ }^{-1-}$ compared with wild-type mice after injury). We speculate that the greater number of nuclei within myofibers of Icaml ${ }^{-1-}$ compared with wild-type mice after injury reflects a compensatory increase in satellite cell/myoblast fusion to account for mispositioned/poorly organized nuclei, as well as impairments in myofiber fusion. If true, the compensatory increase in satellite cell/myoblast fusion resulting from the absence of ICAM-1 failed to restore protein synthesis and myofiber size to wild-type levels after injury.

The premise for nuclear positioning/organization serving as a driver of phenotypic changes within myofibers after injury is rooted in intuition, as the extent to which transcription and other nuclear activities (eg, nuclear import and export) are influenced by their position/organization within myofibers has yet to be established. Newlands et $\mathrm{al}^{59}$ reported that all nuclei within nuclear chains after muscle injury are transcriptionally competent and that some, but not all, of them are actively transcribing muscle-specific genes. The transcriptional competence and activity of nuclei that are clustered together after muscle injury, however, remain to be determined. As functional activities of nuclei within myotubes/myofibers are heterogeneous, as well as temporally, spatially, and developmentally regulated, ${ }^{59-62}$ it is conceivable that nuclear positioning and organization influences the hypertrophy phenotype of myofibers after muscle injury.

In the present study, the greater number of nuclei within myofibers of Icam $1^{-1-}$ mice after injury was accompanied with impairments in the restoration of myofiber size, protein synthesis, and nuclear positioning/organization. Regression analysis demonstrated that increases in myofiber size after injury were less responsive to myonuclear accretion in Icam $1^{-1-}$ compared with wild-type mice. Thus, the absence of ICAM-1 uncoupled the temporal and statistical relationship between myonuclear accretion and indexes of myofiber hypertrophy after injury. To reconcile these findings, we speculate that ICAM-1 facilitates myofiber hypertrophy after injury by regulating processes that follow myogenic cell fusion/myonuclear accretion, such as gene transcription and translation, which we suspect is influenced by the position/organization of nuclei within myofibers. Clearly, additional work is needed to identify mechanisms through which ICAM-1 facilitates protein synthesis and myofiber hypertrophy after injury.

A critical barrier to the development of new approaches for treating musculoskeletal injuries is the lack of knowledge of how the local inflammatory response regulates muscle regeneration. The results reported herein reduce that barrier by demonstrating that myogenic cell expression of ICAM-1 contributes to regenerating and hypertrophic processes within skeletal muscle after injury. A defined role of myogenic cell expression of ICAM-1 in muscle regeneration after injury, however, awaits future studies that manipulate ICAM-1 expression only in myogenic cells.

\section{Author Contributions}

R.A.M. and K.H.B. designed experiments, acquired, analyzed, interpreted, and presented data; R.A.M. wrote the manuscript; D.C.M. acquired, analyzed, and interpreted data; B.M.R. acquired, analyzed, interpreted, and presented data; A.N.S. analyzed images of single myofibers; S.L.D. analyzed protein synthesis; R.W. designed experiments, and acquired, analyzed, interpreted, and presented data; and F.X.P. conceived of the study and designed experiments, acquired, analyzed, interpreted, and presented data, and wrote the manuscript.

\section{References}

1. Novak ML, Koh TJ: Phenotypic transitions of macrophages orchestrate tissue repair. Am J Pathol 2013, 183:1352-1363 
2. Pizza FX: Neutrophils and macrophages in muscle damage and repair. Edited by Tiidus PM. In Skeletal Muscle Damage and Repair. Champaign, IL: Human Kinetics, 2008. pp. 49-57

3. Saclier M, Cuvellier S, Magnan M, Mounier R, Chazaud B: Monocyte/macrophage interactions with myogenic precursor cells during skeletal muscle regeneration. FEBS J 2013, 280:4118-4130

4. Dumont NA, Bentzinger CF, Sincennes MC, Rudnicki MA: Satellite cells and skeletal muscle regeneration. Compr Physiol 2015, 5:1027-1059

5. DiPasquale DM, Cheng M, Billich W, Huang SA, van Rooijen N, Hornberger TA, Koh TJ: Urokinase-type plasminogen activator and macrophages are required for skeletal muscle hypertrophy in mice. Am J Physiol Cell Physiol 2007, 293:C1278-C1285

6. Arnold L, Henry A, Poron F, Baba-Amer Y, van Rooijen N, Plonquet A, Gherardi RK, Chazaud B: Inflammatory monocytes recruited after skeletal muscle injury switch into antiinflammatory macrophages to support myogenesis. J Exp Med 2007, 204:1057-1069

7. Novak ML, Weinheimer-Haus EM, Koh TJ: Macrophage activation and skeletal muscle healing following traumatic injury. J Pathol 2014, 232:344-355

8. Bencze M, Negroni E, Vallese D, Yacoub-Youssef H, Chaouch S, Wolff A, Aamiri A, Di Santo JP, Chazaud B, Butler-Browne G, Savino W, Mouly V, Riederer I: Proinflammatory macrophages enhance the regenerative capacity of human myoblasts by modifying their kinetics of proliferation and differentiation. Mol Ther 2012, 20: $2168-2179$

9. Tonkin J, Temmerman L, Sampson RD, Gallego-Colon E, Barberi L, Bilbao D, Schneider MD, Musaro A, Rosenthal N: Monocyte/macrophage-derived IGF-1 orchestrates murine skeletal muscle regeneration and modulates autocrine polarization. Mol Ther 2015, 23:1189-1200

10. Bryer SC, Fantuzzi G, Van Rooijen N, Koh TJ: Urokinase-type plasminogen activator plays essential roles in macrophage chemotaxis and skeletal muscle regeneration. J Immunol 2008, 180:1179-1188

11. Rigamonti E, Touvier T, Clementi E, Manfredi AA, Brunelli S, Rovere-Querini P: Requirement of inducible nitric oxide synthase for skeletal muscle regeneration after acute damage. J Immunol 2013, 190:1767-1777

12. Zhang C, Li Y, Wu Y, Wang L, Wang X, Du J: Interleukin-6/signal transducer and activator of transcription 3 (STAT3) pathway is essential for macrophage infiltration and myoblast proliferation during muscle regeneration. J Biol Chem 2013, 288:1489-1499

13. Dearth CL, Goh Q, Marino JS, Cicinelli PA, Torres-Palsa MJ, Pierre P, Worth RG, Pizza FX: Skeletal muscle cells express ICAM-1 after muscle overload and ICAM-1 contributes to the ensuing hypertrophic response. PLoS One 2013, 8:e58486

14. Marino JS, Tausch BJ, Dearth CL, Manacci MV, McLoughlin TJ, Rakyta SJ, Linsenmayer MP, Pizza FX: Beta2-integrins contribute to skeletal muscle hypertrophy in mice. Am J Physiol, Cell Physiol 2008, 295:C1026-C1036

15. Du H, Shih CH, Wosczyna MN, Mueller AA, Cho J, Aggarwal A, Rando TA, Feldman BJ: Macrophage-released ADAMTS1 promotes muscle stem cell activation. Nat Commun 2017, 8:669

16. Pavlath GK: Current Progress Towards Understanding Mechanisms of Myoblast Fusion in Mammals. Berlin, Germany, Springer-Verlag Berlin, 2011

17. Krauss RS, Cole F, Gaio U, Takaesu G, Zhang W, Kang JS: Close encounters: regulation of vertebrate skeletal myogenesis by cell-cell contact. J Cell Scie 2005, 118:2355-2362

18. Rahman A, Fazal F: Hug tightly and say goodbye: role of endothelial ICAM-1 in leukocyte transmigration. Antioxid Redox Signal 2009, 11: 823-839

19. Lawson C, Wolf S: ICAM-1 signaling in endothelial cells. Pharmacol Rep 2009, 61:22-32

20. Torres-Palsa MJ, Koziol MV, Goh Q, Cicinelli PA, Peterson JM, Pizza FX: Expression of intercellular adhesion molecule-1 by myofibers in mdx mice. Muscle Nerve 2015, 52:795-802
21. Goh Q, Dearth CL, Corbett JT, Pierre P, Chadee DN, Pizza FX: Intercellular adhesion molecule-1 expression by skeletal muscle cells augments myogenesis. Exp Cell Res 2015, 331:292-308

22. Pizza FX, Martin RA, Springer EM, Leffler MS, Woelmer BR, Recker IJ, Leaman DW: Intercellular adhesion molecule-1 augments myoblast adhesion and fusion through homophilic trans-interactions. Sci Rep 2017, 7:5094

23. Marino M, Scuderi F, Mazzarelli P, Mannella F, Provenzano C, Bartoccioni E: Constitutive and cytokine-induced expression of MHC and intercellular adhesion molecule-1 (ICAM-1) on human myoblasts. J Neuroimmunol 2001, 116:94-101

24. Marino M, Scuderi F, Mannella F, Bartoccioni E: TGF-beta 1 and IL10 modulate IL- 1 beta-induced membrane and soluble ICAM-1 in human myoblasts. J Neuroimmunol 2003, 134:151-157

25. Goebels N, Michaelis D, Wekerle H, Hohlfeld R: Human myoblasts as antigen-presenting cells. J Immunol 1992, 149:661-667

26. Michaelis D, Goebels N, Hohlfeld R: Constitutive and cytokineinduced expression of human leukocyte antigens and cell adhesion molecules by human myotubes. Am J Pathol 1993, 143:1142-1149

27. De Bleecker JL, Engel AG: Expression of cell adhesion molecules in inflammatory myopathies and Duchenne dystrophy. J Neuropathol Exp Neurol 1994, 53:369-376

28. Tews DS, Goebel HH: Expression of cell adhesion molecules in inflammatory myopathies. J Neuroimmunol 1995, 59:185-194

29. Bartoccioni E, Gallucci S, Scuderi F, Ricci E, Servidei S, Broccolini A, Tonali P: MHC class I, MHC class II and intercellular adhesion molecule-1 (ICAM-1) expression in inflammatory myopathies. Clin Exp Immunol 1994, 95:166-172

30. Robker RL, Collins RG, Beaudet AL, Mersmann HJ, Smith CW: Leukocyte migration in adipose tissue of mice null for ICAM-1 and Mac-1 adhesion receptors. Obes Res 2004, 12:936-940

31. Murphy MM, Lawson JA, Mathew SJ, Hutcheson DA, Kardon G: Satellite cells, connective tissue fibroblasts and their interactions are crucial for muscle regeneration. Development 2011, 138:3625-3637

32. Prigge JR, Wiley JA, Talago EA, Young EM, Johns LL, Kundert JA, Sonsteng KM, Halford WP, Capecchi MR, Schmidt EE: Nuclear double-fluorescent reporter for in vivo and ex vivo analyses of biological transitions in mouse nuclei. Mamm Genome 2013, 24: 389-399

33. Minamide LS, Bamburg JR: A filter paper dye-binding assay for quantitative determination of protein without interference from reducing agents or detergents. Anal Biochem 1990, 190:66-70

34. Schmidt EK, Clavarino G, Ceppi M, Pierre P: SUnSET, a nonradioactive method to monitor protein synthesis. Nat Methods 2009, 6: $275-277$

35. Goodman CA, Mabrey DM, Frey JW, Miu MH, Schmidt EK, Pierre P, Hornberger TA: Novel insights into the regulation of skeletal muscle protein synthesis as revealed by a new nonradioactive in vivo technique. FASEB J 2011, 25:1028-1039

36. Maesner CC, Almada AE, Wagers AJ: Established cell surface markers efficiently isolate highly overlapping populations of skeletal muscle satellite cells by fluorescence-activated cell sorting. Skelet Muscle 2016, 6:35

37. Sacco A, Doyonnas R, Kraft P, Vitorovic S, Blau HM: Self-renewal and expansion of single transplanted muscle stem cells. Nature 2008, 456:502-506

38. Corona BT, Henderson BE, Ward CL, Greising SM: Contribution of minced muscle graft progenitor cells to muscle fiber formation after volumetric muscle loss injury in wild-type and immune deficient mice. Physiol Rep 2017, 5:e13249

39. Mintz EL, Passipieri JA, Lovell DY, Christ GJ: Applications of in vivo functional testing of the rat tibialis anterior for evaluating tissue engineered skeletal muscle repair. J Vis Exp 2016, (116):54487

40. Brack AS, Bildsoe H, Hughes SM: Evidence that satellite cell decrement contributes to preferential decline in nuclear number from large fibres during murine age-related muscle atrophy. J Cell Sci 2005, 118:4813-4821 
41. Chan S, Head SI: The role of branched fibres in the pathogenesis of Duchenne muscular dystrophy. Exp Physiol 2011, 96:564-571

42. Pichavant C, Pavlath GK: Incidence and severity of myofiber branching with regeneration and aging. Skelet Muscle 2014, 4:9

43. Griffin CA, Kafadar KA, Pavlath GK: MOR23 promotes muscle regeneration and regulates cell adhesion and migration. Dev Cell 2009, 17:649-661

44. Schmalbruch H: The morphology of regeneration of skeletal muscles in the rat. Tissue Cell 1976, 8:673-692

45. Wada K, Katsuta S, Soya H: Formation process and fate of the nuclear chain after injury in regenerated myofiber. Anat Rec (Hoboken) 2008, 291:122-128

46. Hardy D, Besnard A, Latil M, Jouvion G, Briand D, Thepenier C, Pascal Q, Guguin A, Gayraud-Morel B, Cavaillon JM, Tajbakhsh S, Rocheteau P, Chretien F: Comparative study of injury models for studying muscle regeneration in mice. PLoS One 2016, 11:e0147198

47. Dumont NA, Wang YX, von Maltzahn J, Pasut A, Bentzinger CF, Brun CE, Rudnicki MA: Dystrophin expression in muscle stem cells regulates their polarity and asymmetric division. Nat Med 2015, 21 : $1455-1463$

48. Kaufman SJ, Foster RF: Replicating myoblasts express a musclespecific phenotype. Proc Natl Acad Sci U S A 1988, 85: 9606-9610

49. Seale P, Sabourin LA, Girgis-Gabardo A, Mansouri A, Gruss P, Rudnicki MA: Pax7 is required for the specification of myogenic satellite cells. Cell 2000, 102:777-786

50. Krauss RS: Regulation of promyogenic signal transduction by cellcell contact and adhesion. Exp Cell Res 2010, 316:3042-3049

51. De Paepe B, De Bleecker JL: Cytokines and chemokines as regulators of skeletal muscle inflammation: presenting the case of Duchenne muscular dystrophy. Mediators Inflamm 2013, 2013: 540370
52. Warren GL, Hulderman T, Jensen N, McKinstry M, Mishra M, Luster MI, Simeonova PP: Physiological role of tumor necrosis factor alpha in traumatic muscle injury. FASEB J 2002, 16 : $1630-1632$

53. Cheng M, Nguyen MH, Fantuzzi G, Koh TJ: Endogenous interferongamma is required for efficient skeletal muscle regeneration. Am J Physiol Cell Physiol 2008, 294:C1183-C1191

54. Horsley V, Jansen KM, Mills ST, Pavlath GK: IL-4 acts as a myoblast recruitment factor during mammalian muscle growth. Cell 2003, 113:483-494

55. Bruusgaard JC, Liestol K, Ekmark M, Kollstad K, Gundersen K: Number and spatial distribution of nuclei in the muscle fibres of normal mice studied in vivo. J Physiol 2003, 551:467-478

56. Folker ES, Baylies MK: Nuclear positioning in muscle development and disease. Front Physiol 2013, 4:363

57. Cadot B, Gache V, Gomes ER: Moving and positioning the nucleus in skeletal muscle: one step at a time. Nucleus 2015, 6:373-381

58. Roman W, Martins JP, Carvalho FA, Voituriez R, Abella JVG, Santos NC, Cadot B, Way M, Gomes ER: Myofibril contraction and crosslinking drive nuclear movement to the periphery of skeletal muscle. Nat Cell Biol 2017, 19:1189-1201

59. Newlands S, Levitt LK, Robinson CS, Karpf AB, Hodgson VR, Wade RP, Hardeman EC: Transcription occurs in pulses in muscle fibers. Genes Dev 1998, 12:2748-2758

60. Pavlath GK, Rich K, Webster SG, Blau HM: Localization of muscle gene products in nuclear domains. Nature 1989, 337:570-573

61. Cutler AA, Jackson JB, Corbett AH, Pavlath GK: Non-equivalence of nuclear import among nuclei in multinucleated skeletal muscle cells. J Cell Sci 2018, 131:jcs207670

62. Ralston E, Hall ZW: Intracellular and surface distribution of a membrane protein (CD8) derived from a single nucleus in multinucleated myotubes. J Cell Biol 1989, 109:2345-2352 